\title{
Mixed Convection in a Two-Sided and Four-Sided Lid-Driven Square Porous Cavity
}

\author{
Shobha Bagai ${ }^{*}$, Manoj Kumar ${ }^{2}$, Arvind Patel $^{2}$ \\ ${ }^{1}$ Cluster Innovation Centre, 3rd Floor University Stadium, G C Narang Road, University of Delhi, Delhi 110007, India \\ ${ }^{2}$ Department of Mathematics, Faculty of Mathematical Sciences, University of Delhi, Delhi 110007, India
}

Corresponding Author Email: mkumar@maths.du.ac.in

https://doi.org/10.18280/ijht.390305

Received: 20 August 2019

Accepted: 19 March 2021

\section{Keywords:}

alternating-direction-implicit (ADI) method, finite difference method, mixed convection, two-sided and four-sided lid-driven flow, porous media

\begin{abstract}
The present paper investigates the mixed convection in a two-sided and four-sided liddriven square cavity in porous media. In the two-sided porous cavity, the left and right walls of the enclosure are maintained at constant but different temperatures, while the top and bottom walls are adiabatic. The top and the bottom walls of the enclosure move with a constant speed from left to right. In the four-sided porous cavity, the top and the bottom walls of the enclosure move from left to right and right to left, respectively, while the left and the right walls move from top to bottom and bottom to top, respectively, with a constant speed. The left and right walls of the enclosure are maintained at different heat fluxes, while the top and bottom walls are maintained at hot and cold temperatures, respectively. The governing equations are discretized by the fully implicit finite difference method, namely, Alternating-Direction-Implicit (ADI) method. The numerical results are analyzed for the effect of Darcy number $(D a=0.001,0.01)$, Prandtl number $(\operatorname{Pr}=7)$, Grashof number $(G r=50,000)$, porosity $(\varepsilon=0.2)$ and viscosity ratio $(\Lambda=1,3)$. The stability and convergence of the considered problem have been proved using the Matrix method.
\end{abstract}

\section{INTRODUCTION}

During the past few decades, the problem of natural or mixed convection in lid-driven square or rectangular cavity with porous media has been widely studied due to its simple geometrical settings and its practical applications [1] such as nuclear waste disposal, coal and grain storage, textile materials, geothermal systems, biological processes, and many others. Mixed convection in lid-driven square or rectangular cavity has various applications in engineering science [2], such as lubricant technology, chemical processing, cooling of microprocessors and electronic components, float glass production, etc. The lid-driven cavity in porous media whose all four walls are kept at different heat flux or temperatures has been studied by many researchers. Mixed or natural convection in a square cavity with three different cases, i.e. (a) all walls of the cavity are kept stationary, (b) one side of the wall is in motion (c) two sides of the cavity are in motion, has been studied in the following literature.

Mixed convection in a square cavity with all walls at rest has been studied by Venkatachalappa et al. [1], Saeid and Mohamad [3], Mansour et al. [4], Basak et al. [5], and Badruddin et al. [6]. Venkatachalappa et al. [1] investigated natural convection inside a square porous cavity using the finite-difference ADI method. Saeid and Mohamad [3] studied the natural convection within the square cavity by keeping its right wall at hot temperature and sinusoidal condition on its left wall. In contrast, the top and bottom walls are adiabatic. Mansour et al. [4] investigated the numerical study of natural convection with thermal radiation inside a wavy porous cavity. Basak et al. [5] examined the natural convection flow inside a square cavity keeping its top wall adiabatic. In contrast, the bottom wall is maintained at hot temperature or a sinusoidal boundary condition. The left and right wall of the cavity is maintained at a cold temperature. They have obtained numerical results for various parameters; Rayleigh number $\left(10^{3} \leq R a \leq 10^{6}\right)$, Darcy number $\left(10^{-5} \leq D a \leq 10^{-3}\right)$, and Prandtl number $(0.71 \leq \operatorname{Pr} \leq 10)$. Badruddin et al. [6] examined the heat transfer by convection, conduction, and radiation using a non-equilibrium thermal model inside a square porous cavity. The numerical results are discussed for various parameters like Rayleigh number, inter-phase heat transfer coefficient radiation, and modified conductivity ratio in terms of Nusselt number for solid and fluid.

Natural or mixed convection in the one-sided lid-driven square porous cavity has been studied by Chattopadhyay and Pandit [7], Kandaswamy et al. [8], Mohan and Satheesh [9], Md. Hidayathulla Khan et al. [10]. Chattopadhyay and Pandit [7] have used the higher-order compact (HOC) scheme to analyze the mixed convection in a lid-driven trapezoidal porous enclosure whose top wall is kept at a motion from left to right. The effect of convection in a trapezoidal porous enclosure is examined for different Peclet numbers. Kandaswamy et al. [8] have numerically investigated the effect of Prandtl number on mixed convection in a one-sided lid-driven square cavity filled with porous media. They have found that conduction is dominated at low Prandtl numbers, while mixed and forced convection dominates the temperature field as the Prandtl number increases. Mohan and Satheesh [9] investigated the double-diffusive mixed convection with magnetohydrodynamic effect in a one-sided lid-driven porous cavity. They have examined the fluid flow in the top-sided liddriven square cavity in both directions with a constant velocity. They analyzed streamline contours, concentration, temperature gradients, and velocity components for a wide range of non-dimensional parameters like Hartmann $(1 \leq \mathrm{Ha}$ 
$\leq 25)$, Lewis $(1 \leq L e \leq 50)$, Prandtl $(P r=0.7)$, Richardson $(R i$ $=1.0)$, Darcy $(D a=1.0)$, and Reynolds $(R e=100)$ numbers. Khan et al. [10] have used the Marker and Cell (MAC) method to analyze the mixed convection in a one-sided lid-driven porous cavity.

Natural or mixed convection inside a two-sided lid-driven porous cavity has been studied by Vardhana and Das [11], Muthtamilselvan et al. [12], Behzadi et al. [13], Mittal et al. [14], Chattopadhyay et al. [15]. They have all investigated the mixed convection inside a two-sided lid-driven square porous cavity, in which the left and right walls move at a constant speed in the same or opposite directions. The left and right walls of the cavity are kept at constant but different temperatures, while the top and bottom walls are adiabatic. Muthtamilselvan et al. [12] carried out numerical computation in a square porous cavity whose top and bottom walls move in opposite directions. Behzadi et al. [13] have investigated the effect of porous media on mixed convection inside a ventilated square cavity, in which an external flow enters the enclosure from a port on the left vertical wall and leaves from a port on the right wall. Md. Hidayathulla Khan et al. [16] investigated the mixed convection under the effect of radiation inside a porous square cavity whose top and bottom walls are kept at a constant temperature, while some portions of the left and right walls of the cavity are partially heated. Vusala and Kumar [17], and Bagai et al. [18, 19] have used the stream functionvorticity formulation to investigate the mixed convection problem with heat or mass transfer in a two-sided or four-sided lid-driven square porous or without a porous cavity.

The present study analyzes the mixed convection in a twosided and four-sided lid-driven square cavity filled with porous media. The governing equations for the considered problem are discretized by a fully implicit finite difference scheme, namely Alternating-Direction-Implicit (ADI) method. We shall also prove the stability and convergence of the numerical scheme to obtain the desired accuracy by the Matrix method.

\section{MATHEMATICAL FORMULATION}

\subsection{Physical description}

The physical model of a two-sided and four-sided lid-driven square cavity of unit size with heat transfer is illustrated in Figure 1. The cavity is filled with homogeneous, isotropic, saturated, sparsely packed porous material of high permeability $K$. The fluid's thermo-physical properties are presumed to be constant, except the density, which varies linearly with temperature as, $\rho=\rho_{0}\left[1-\beta\left(T_{h}-T_{c}\right)\right]$, where $\beta$ is the thermal expansion coefficient, and the subscript 0 denotes the reference state. Natural convection occurs in the cavity due to temperature gradient, and forced convection occurs due to the motions of the lids. Thus, the combination of natural convection and forced convection results in a mixed convection problem.

In the two-sided porous cavity, the top and the bottom walls of the cavity move from left to right with velocity $u=1$. The left and right walls of the porous cavity are maintained at cold $(T=-1)$ and hot $(T=1)$ temperatures, respectively, while the top and the bottom walls of the cavity are adiabatic. In a foursided cavity, the top and the bottom walls of the cavity move in opposite directions with velocity $u=1$. The left and right walls of the cavity move with velocity $v=1$ in the opposite directions. The top and the bottom walls of the porous cavity are maintained at hot $(T=1)$ and cold $(T=-1)$ temperatures, respectively, while the left and right walls of the cavity are kept with heat flux $\partial T / \partial x=-1$ and $\partial T / \partial x=1$ respectively.

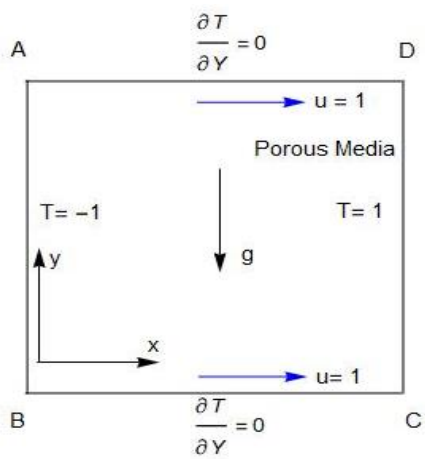

(a) Two-sided lid-driven square porous cavity

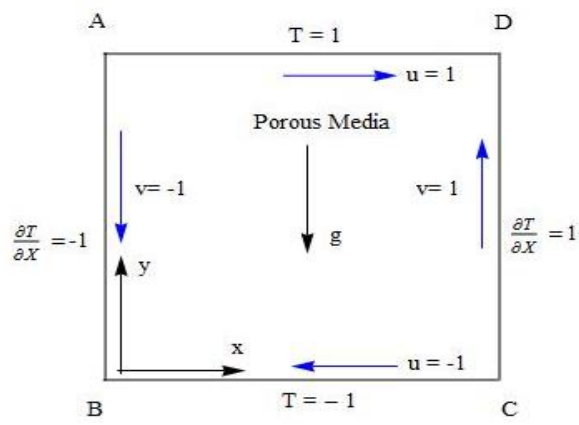

(b) Four-sided lid-driven square porous cavity

Figure 1. Flow configuration and coordinate system for twosided and four-sided lid-driven square cavity in the porous media

\subsection{Governing equations}

The governing equations of mixed convection in a liddriven square porous cavity based on Boussinesq approximations, using the stream function-vorticity $(\psi-\xi)$ formulation in the non-dimensional form [1] can be expressed as:

$$
\begin{gathered}
\frac{1}{\varepsilon} \frac{\partial \xi}{\partial t}+\frac{1}{\varepsilon^{2}} u \frac{\partial \xi}{\partial x}+\frac{1}{\varepsilon^{2}} v \frac{\partial \xi}{\partial y}=\frac{G r}{2} \frac{\partial T}{\partial y}+ \\
\Lambda\left(\frac{\partial^{2} \xi}{\partial x^{2}}+\frac{\partial^{2} \xi}{\partial y^{2}}\right)-\frac{1}{D a} \xi \\
S \frac{\partial T}{\partial t}+u \frac{\partial T}{\partial x}+v \frac{\partial T}{\partial y}=\frac{1}{P r}\left(\frac{\partial^{2} T}{\partial x^{2}}+\frac{\partial^{2} T}{\partial y^{2}}\right), \\
\nabla^{2} \psi=-\xi \\
u=\frac{\partial \psi}{\partial y}, v=-\frac{\partial \psi}{\partial x} .
\end{gathered}
$$

where,

$$
\begin{aligned}
& \xi=\frac{\partial v}{\partial x}-\frac{\partial u}{\partial y}, x=\frac{x^{\prime}}{L}, y=\frac{y^{\prime}}{L}, u=\frac{u^{\prime}}{\alpha / L}, v=\frac{v^{\prime}}{\alpha / L}, t=\frac{t^{\prime}}{\alpha^{2} / L} \\
& \xi=\frac{\omega}{\alpha^{2} / L}, \psi=\frac{\psi^{\prime}}{L}, T=\frac{\theta-\theta_{0}}{\theta_{h}-\theta_{c}}, \theta=\frac{\theta_{h}+\theta_{c}}{2}, \nabla^{2}=\frac{\partial^{2}}{\partial x^{2}}+\frac{\partial^{2}}{\partial y^{2}}
\end{aligned}
$$


The non-dimensional parameters are Prandtl number, Darcy number, viscosity ratio and Grashof number, defined as follows:

$$
\operatorname{Pr}=\frac{v}{\alpha}, D a=\frac{K}{L^{2}}, \Lambda=\frac{\mu^{\prime}}{\mu}, G r=\frac{g \beta\left(\theta_{h}-\theta_{c}\right) L^{3}}{v^{2}} .
$$

The Darcy number is the ratio of permeability of the porous media to square of the length of the cavity. The medium's permeability is defined as, $K=D_{p}^{2} \varepsilon^{2} / 144(1-\varepsilon)^{2}$, where $D_{p}$ is the particle diameter and $\varepsilon$ its porosity. The porosity in the porous media structure is an important indicator, which directly affects the movement characteristics of the fluid. So, its effect can be considered by varying the Darcy number.

The initial and the boundary conditions associated with the system (1) to (4) are:

\section{Case 1: Two-sided lid-driven porous cavity}

$$
\begin{gathered}
t=0 ; u=v=T=\psi=\xi=0,0 \leq x, y \leq 1 \\
t>0 ; x=0: T=-1, \psi=\frac{\partial \psi}{\partial x}=0(i . e \cdot v=0) \\
x=1: T=1, \psi=\frac{\partial \psi}{\partial x}=0(i . e \cdot v=0) \\
y=0,1: \frac{\partial T}{\partial y}=0, \psi=0, \frac{\partial \psi}{\partial y}=1(i . e \cdot u=1) .
\end{gathered}
$$

\section{Case 2: Four-sided lid-driven porous cavity}

$$
\begin{gathered}
t=0 ; u=v=T=\psi=\xi=0, \quad 0 \leq x, y \leq 1, \\
t>0 ; x=0: \frac{\partial T}{\partial x}=-1, \psi=0, \frac{\partial \psi}{\partial x}=1(i . e \cdot v=-1), \\
x=1: \frac{\partial T}{\partial x}=1, \psi=0, \frac{\partial \psi}{\partial x}=-1(i . e \cdot v=1), \\
y=0: T=-1, \psi=0, \frac{\partial \psi}{\partial y}=-1(\text { i.e. } u=-1), \\
y=1: T=1, \psi=0, \frac{\partial \psi}{\partial y}=1(\text { i.e.u } u) .
\end{gathered}
$$

Heat transfer along the left and right walls of the cavity is also studied with the help of the average Nusselt number. The local and average Nusselt numbers along the left and right wall of the enclosure are defined as follows:

\begin{tabular}{c|c|c}
\hline & Left wall & Right wall \\
\hline $\begin{array}{c}\text { Local Nusselt } \\
\text { number }\end{array}$ & $N u_{l}=\left.\frac{\partial \mathrm{T}}{\partial \mathrm{x}}\right|_{x=0}$ & $N u_{r}=\left.\frac{\partial \mathrm{T}}{\partial \mathrm{x}}\right|_{x=1}$ \\
\hline $\begin{array}{c}\text { Average Nusselt } \\
\text { number }\end{array}$ & $\overline{N u_{l}}=\int_{0}^{1} N u_{l} \mathrm{dy}$ & $\overline{N u_{r}}=\int_{0}^{1} N u_{r} \mathrm{dy}$ \\
\hline
\end{tabular}

\section{NUMERICAL DISCRETIZATION}

The finite difference method is used to discretize the Poisson, vorticity, and energy transport equations. The coupled Eqns. (1) to (3) are solved numerically utilizing Alternating-Direction-Implicit (ADI) method. Using the ADI method, we obtain a system of tri-diagonal equations, which is solved by LU-decomposition for vorticity $\xi$ and temperature $T$. The second-order partial derivatives of flow variable $\psi$ in Eq. (3) are discretized by the second-order central finite difference quotients.
The first and second-order partial derivatives of stream function $\psi$, vorticity $\xi$, temperature $T$ in Eqns. (1) to (3) with respect to time and space variables are discretized using forward-time and central-space (FTCS) finite-difference quotients for the ADI Scheme [17, 20]. The finite difference quotients are used to discretize Eqns. (1) to (3) to get the following discretized equations.

In view of the discretization Eq. (3) can be written as:

$$
\begin{gathered}
\frac{\psi_{i+1, j}^{t+1}-2 \psi_{i, j}^{t+1}+\psi_{i-1, j}^{t+1}}{\Delta x^{2}} \\
+\frac{\psi_{i, j+1}^{t+1}-2 \psi_{i, j}^{t+1}+\psi_{i, j-1}^{t+1}}{\Delta y^{2}}=-\xi_{i, j}^{t+1},
\end{gathered}
$$

The discretized Eq. (1) with time step $t+\frac{1}{2}$ and $t+1$ can be expressed as:

$$
\begin{aligned}
& {\left[\frac{\Delta t}{8 \varepsilon \Delta x \Delta y}\left(\psi_{i+1, j}^{t}-\psi_{i-1, j}^{t}\right)-\frac{\varepsilon \Lambda \Delta t}{2 \Delta y^{2}}\right] \xi_{i, j-1}^{t+\frac{1}{2}}+\left[1+\frac{\varepsilon \Lambda \Delta t}{\Delta y^{2}}\right] \xi_{i, j}^{t+\frac{1}{2}}+} \\
& {\left[-\frac{\Delta t}{8 \varepsilon \Delta x \Delta y}\left(\psi_{i+1, j}^{t}-\psi_{i-1, j}^{t}\right)-\frac{\varepsilon \Lambda \Delta t}{2 \Delta y^{2}}\right] \xi_{i, j+1}^{t+\frac{1}{2}}=} \\
& {\left[\frac{\Delta t}{8 \varepsilon \Delta x \Delta y}\left(\psi_{i, j+1}^{t}-\psi_{i, j-1}^{t}\right)+\frac{\varepsilon \Lambda \Delta t}{2 \Delta x^{2}}\right] \xi_{i-1, j}^{t}+\left[1-\frac{\varepsilon \Lambda \Delta t}{\Delta x^{2}}-\frac{\varepsilon \Delta t}{2 D a}\right] \xi_{i, j}^{t}+} \\
& {\left[-\frac{\Delta t}{8 \varepsilon \Delta x \Delta y}\left(\psi_{i, j+1}^{t}-\psi_{i, j-1}^{t}\right)+\frac{\varepsilon \Lambda \Delta t}{2 \Delta x^{2}}\right] \xi_{i+1, j}^{t}+\frac{\varepsilon G r \Delta t}{8 \Delta y}\left[T_{i, j+1}^{t}-T_{i, j-1}^{t}\right]}
\end{aligned}
$$

and,

$$
\begin{aligned}
& {\left[-\frac{\Delta t}{8 \varepsilon \Delta x \Delta y}\left(\psi_{i, j+1}^{t}-\psi_{i, j-1}^{t}\right)-\frac{\varepsilon \Lambda \Delta t}{2 \Delta x^{2}}\right] \xi_{i-1, j}^{t+1}+\left[1+\frac{\varepsilon \Lambda \Delta t}{\Delta x^{2}}\right] \xi_{i, j}^{t+1}} \\
& +\left[\frac{\Delta t}{8 \varepsilon \Delta x \Delta y}\left(\psi_{i, j+1}^{t}-\psi_{i, j-1}^{t}\right)-\frac{\varepsilon \Lambda \Delta t}{2 \Delta x^{2}}\right] \xi_{i+1, j}^{t+1}= \\
& {\left[-\frac{\Delta t}{8 \varepsilon \Delta x \Delta y}\left(\psi_{i+1, j}^{t}-\psi_{i-1, j}^{t}\right)+\frac{\varepsilon \Lambda \Delta t}{2 \Delta y^{2}}\right] \xi_{i, j-1}^{t+\frac{1}{2}}+\left[1-\frac{\varepsilon \Lambda \Delta t}{\Delta y^{2}}-\frac{\varepsilon \Delta t}{2 D a}\right] \xi_{i, j}^{t+\frac{1}{2}}} \\
& +\left[\frac{\Delta t}{8 \varepsilon \Delta x \Delta y}\left(\psi_{i+1, j}^{t}-\psi_{i-1, j}^{t}\right)+\frac{\varepsilon \Lambda \Delta t}{2 \Delta y^{2}}\right] \xi_{i, j+1}^{t+\frac{1}{2}}+\frac{\varepsilon G r \Delta t}{8 \Delta y}\left[T_{i, j+1}^{t}-T_{i, j-1}^{t}\right]
\end{aligned}
$$

The Eq. (2) at time step $t+\frac{1}{2}$ and $t+1$ can be discretized using the ADI scheme as follows:

$$
\begin{aligned}
& {\left[\frac{\Delta t}{8 S \Delta x \Delta y}\left(\psi_{i+1, j}^{t}-\psi_{i-1, j}^{t}\right)-\frac{\Delta t}{2 S \operatorname{Pr} \Delta y^{2}}\right] T_{i, j-1}^{t+\frac{1}{2}}+\left[1+\frac{\Delta t}{\operatorname{SPr} \Delta y^{2}}\right] T_{i, j}^{t+\frac{1}{2}}+} \\
& {\left[-\frac{\Delta t}{8 S \Delta x \Delta y}\left(\psi_{i+1, j}^{t}-\psi_{i-1, j}^{t}\right)-\frac{\Delta t}{2 S \operatorname{Pr} \Delta y^{2}}\right] T_{i, j+1}^{t+\frac{1}{2}}=\left[\frac{\Delta t}{8 S \Delta x \Delta y}\left(\psi_{i, j+1}^{t}-\psi_{i, j-1}^{t}\right)+\frac{\Delta t}{2 S \operatorname{Pr} \Delta x^{2}}\right] T_{i-1, j}^{t}} \\
& +\left[1-\frac{\Delta t}{\operatorname{SPr} \Delta x^{2}}\right] T_{i, j}^{t}+\left[-\frac{\Delta t}{8 S \Delta x \Delta y}\left(\psi_{i, j+1}^{t}-\psi_{i, j-1}^{t}\right)+\frac{\Delta t}{2 \operatorname{SPr} \Delta x^{2}}\right] T_{i+1, j}^{t}
\end{aligned}
$$

and,

$$
\begin{aligned}
& {\left[-\frac{\Delta t}{2 \operatorname{SPr} \Delta x^{2}}-\frac{\Delta t}{8 S \Delta x \Delta y}\left(\psi_{i, j+1}^{t}-\psi_{i, j-1}^{t}\right)\right] T_{i-1, j}^{t+1}+\left[1+\frac{\Delta t}{\operatorname{SPr} \Delta x^{2}}\right] T_{i, j}^{t+1}+} \\
& {\left[-\frac{\Delta t}{2 \operatorname{SPr} \Delta x^{2}}+\frac{\Delta t}{8 S \Delta x \Delta y}\left(\psi_{i, j+1}^{t}-\psi_{i, j-1}^{t}\right)\right] T_{i+1, j}^{t+1}=\left[\frac{\Delta t}{2 \operatorname{SPr} \Delta y^{2}}-\frac{\Delta t}{8 S \Delta x \Delta y}\left(\psi_{i+1, j}^{t}-\psi_{i-1, j}^{t}\right)\right] T_{i, j-1}^{t+\frac{1}{2}}} \\
& +\left[1-\frac{\Delta t}{\operatorname{SPr} \Delta y^{2}}\right] T_{i, j}^{t+\frac{1}{2}}+\left[\frac{\Delta t}{2 \operatorname{SPr} \Delta y^{2}}+\frac{\Delta t}{8 S \Delta x \Delta y}\left(\psi_{i+1, j}^{t}-\psi_{i-1, j}^{t}\right)\right] T_{i, j+1}^{t+\frac{1}{2}}
\end{aligned}
$$




\section{STABILITY AND CONVERGENCE OF THE NUMERICAL METHOD}

This section proves the stability and convergence of the considered problem using the Matrix method. We will adopt the following difference approximation formula for first-order partial derivatives [20]:

$$
\begin{gathered}
D_{(+, x)}(\Delta x) \xi_{\mathrm{i}, \mathrm{j}}=\frac{\xi_{\mathrm{i}+1, \mathrm{j}}-\xi_{\mathrm{i}, \mathrm{j}}}{\Delta x}+O(\Delta x), \\
D_{(-, x)}(\Delta x) \xi_{\mathrm{i}, \mathrm{j}}=\frac{\xi_{\mathrm{i}, \mathrm{j}}-\xi_{\mathrm{i}-1, \mathrm{j}}}{\Delta x}+O(\Delta x), \\
D_{(0, x)}(\Delta x) \xi_{\mathrm{i}, \mathrm{j}}=\frac{\xi_{\mathrm{i}+1, \mathrm{j}}-\xi_{\mathrm{i}-1, \mathrm{j}}}{2 \Delta x}+O\left(\Delta x^{2}\right) .
\end{gathered}
$$

The second-order partial derivatives will be approximated by second-order central difference formula.

$$
D_{(0, x)}^{2}(\Delta x) \xi_{i, j}=\frac{\xi_{i+1, j}-2 \xi_{i, j}+\xi_{i-1, j}}{\Delta x^{2}}+O\left(\Delta x^{2}\right),
$$

where, $\Delta x$ is the step length in $\mathrm{x}$-direction. Similar formula will be adopted for $y$-direction. Let $\Delta t$ denotes the step length in time $(t)$-direction. Further, we assume $A^{*}=D^{2}{ }_{0, x}, B^{*}=$ $D^{2}{ }_{0, y}, C^{*}=D_{0, x}$ and $D^{*}=D_{0, y}$. Eq. (1) at time step $t+\frac{1}{2}$ can be discretized as:

$$
\begin{gathered}
\xi_{i, j}{ }^{t+\frac{1}{2}}-\xi_{i, j}{ }^{t}=-\frac{\Delta t}{2 \varepsilon} u_{i, j} C^{*} \xi_{i, j}{ }^{t}-\frac{\Delta t}{2 \varepsilon} v_{i, j} D^{*} \xi_{i, j}{ }^{t+\frac{1}{2}} \\
+\frac{\varepsilon \Delta t}{4} G r D^{*} T_{i, j}{ }^{t}+\frac{\varepsilon \Lambda \Delta t}{2} A^{*} \xi_{i, j}{ }^{t} \\
+\frac{\varepsilon \Lambda \Delta t}{2} B^{*} \xi_{i, j}{ }^{t+\frac{1}{2}}-\frac{\varepsilon \Delta t}{2 D a} \xi_{i, j}{ }^{t}
\end{gathered}
$$

On simplification we have:

$$
\begin{aligned}
{[I+} & \left.\frac{\Delta t}{2 \varepsilon} v_{i, j} D^{*}-\frac{\varepsilon \Lambda \Delta t}{2} B^{*}\right] \xi_{i, j}{ }^{t+\frac{1}{2}} \\
& =\left[\frac{\varepsilon \Delta t}{4} G r D^{*}\right] T_{i, j}{ }^{t} \\
& +\left[I-\frac{\Delta t}{2 \varepsilon} u_{i, j} C^{*}+\frac{\varepsilon \Lambda \Delta t}{2} A^{*}-\frac{\varepsilon \Delta t}{2 D a}\right] \xi_{i, j}{ }^{t}
\end{aligned}
$$

This implies,

$$
\begin{gathered}
\xi_{i, j}{ }^{t+\frac{1}{2}}=E_{1} \xi_{i, j}{ }^{t}+F_{1} T_{i, j}{ }^{t} \\
E_{1}=\left[I+\frac{\Delta t}{2 \varepsilon} v_{i, j} D^{*}-\frac{\varepsilon \Lambda \Delta t}{2} B^{*}\right]^{-1}\left[I-\frac{\Delta t}{2 \varepsilon} u_{i, j} C^{*}+\frac{\varepsilon \Lambda \Delta t}{2} A^{*}\right. \\
\left.-\frac{\varepsilon \Delta t}{2 D a}\right] \\
F_{1}=\left[I+\frac{\Delta t}{2 \varepsilon} v_{i, j} D^{*}-\frac{\varepsilon \Lambda \Delta t}{2} B^{*}\right]^{-1}\left[\frac{\varepsilon \Delta t}{4} G r D^{*}\right]
\end{gathered}
$$

Eq. (1) at time step $t+1$ can be discretized as:

$$
\begin{aligned}
\xi_{i, j}{ }^{t+1}-\xi_{i, j}{ }^{t+} & \frac{1}{2} \\
= & -\frac{\Delta t}{2 \varepsilon} u_{i, j} C^{*} \xi_{i, j}{ }^{t+1}-\frac{\Delta t}{2 \varepsilon} v_{i, j} D^{*} \xi_{i, j}{ }^{t+\frac{1}{2}} \\
& +\frac{\varepsilon \Delta t}{4} G r D^{*} T_{i, j}{ }^{t}+\frac{\varepsilon \Lambda \Delta t}{2} A^{*} \xi_{i, j}{ }^{t+1} \\
& +\frac{\varepsilon \Lambda \Delta t}{2} B^{*} \xi_{i, j}{ }^{t+\frac{1}{2}}-\frac{\varepsilon \Delta t}{2 D a} \xi_{i, j}{ }^{t+\frac{1}{2}}
\end{aligned}
$$

Simplifying the above equation, we get:

$$
\begin{aligned}
& {\left[I+\frac{\Delta t}{2 \varepsilon} u_{i, j} C^{*}-\frac{\varepsilon \Lambda \Delta t}{2} A^{*}\right] \xi_{i, j}{ }^{t+1}=\left[\frac{\varepsilon \Delta t}{4} G r D^{*}\right] T_{i, j}{ }^{t}} \\
& +\left[I-\frac{\Delta t}{2 \varepsilon} v_{i, j} D^{*}+\frac{\varepsilon \Lambda \Delta t}{2} B^{*}-\frac{\varepsilon \Delta t}{2 ? D a}\right] \xi_{i, j}{ }^{t+\frac{1}{2}}
\end{aligned}
$$

This implies,

$$
\begin{gathered}
\xi_{i, j}{ }^{t+1}=G_{1} \xi_{i, j}{ }^{t+\frac{1}{2}}+H_{1} T_{i, j}{ }^{t} \\
G_{1}=\left[I+\frac{\Delta t}{2 \varepsilon} u_{i, j} C^{*}-\frac{\varepsilon \Lambda \Delta t}{2} A^{*}\right]^{-1}\left[I-\frac{\Delta t}{2 \varepsilon} v_{i, j} D^{*}+\frac{\varepsilon \Lambda \Delta t}{2} B^{*}\right. \\
\left.-\frac{\varepsilon \Delta t}{2 D a}\right] \\
H_{1}=\left[I+\frac{\Delta t}{2 \varepsilon} u_{i, j} C^{*}-\frac{\varepsilon \Lambda \Delta t}{2} A^{*}\right]^{-1}\left[\frac{\varepsilon \Delta t}{4} G r D^{*}\right]
\end{gathered}
$$

Combining the Eqns. (7) and (8) we obtain,

$$
\xi_{i, j}^{t+1}=G_{1} E_{1} \xi_{i, j}^{t}+\left(G_{1} F_{1}+H_{1}\right) T_{i, j}{ }^{t}
$$

The energy Eq. (2) at time step $t+\frac{1}{2}$ and $t+1$ can be discretized as:

$$
\begin{aligned}
& T_{i, j}^{t+\frac{1}{2}}-T_{i, j}^{t}=\frac{\Delta t}{2 S P r} A^{*} T_{i, j}^{t} \\
& +\frac{\Delta t}{2 S P r} B^{*} T_{i, j}^{t+\frac{1}{2}}-\frac{\Delta t}{2 S} u_{i, j} C^{*} T_{i, j}^{t}-\frac{\Delta t}{2 S} v_{i, j} D^{*} T_{i, j}^{t+\frac{1}{2}} \\
& T_{i, j}^{t+1}-T_{i, j}^{t+\frac{1}{2}}=\frac{\Delta t}{2 S P r} A^{*} T_{i, j}^{t+1} \\
& +\frac{\Delta t}{2 S P r} B^{*} T_{i, j}^{t+\frac{1}{2}}-\frac{\Delta t}{2 S} u_{i, j} C^{*} T_{i, j}^{t+1}-\frac{\Delta t}{2 S} v_{i, j} D^{*} T_{i, j}^{t+\frac{1}{2}}
\end{aligned}
$$

On rearranging, the Eqns. (10) and (11) can be expressed as:

$$
\begin{aligned}
& {\left[I+\frac{\Delta t}{2 S} v_{i, j} D^{*}-\frac{\Delta t}{2 S P r} B^{*}\right] T_{i, j}^{t+\frac{1}{2}}} \\
& =\left[I-\frac{\Delta t}{2 S} u_{i, j} C^{*}+\frac{\Delta t}{2 S P r} A^{*}\right] T_{i, j}^{t} \\
& {\left[I+\frac{\Delta t}{2 S} u_{i, j} C^{*}-\frac{\Delta t}{2 S P r} A^{*}\right] T_{i, j}^{t+1}} \\
& =\left[I-\frac{\Delta t}{2 S} v_{i, j} D^{*}+\frac{\Delta t}{2 S P r} B^{*}\right] T_{i, j}^{t+\frac{1}{2}}
\end{aligned}
$$

Combining Eqns. (12) and (13) we obtain,

$$
\begin{aligned}
& {\left[I+\frac{\Delta t}{2 S} u_{i, j} C^{*}-\frac{\Delta t}{2 S P r} A^{*}\right] T_{i, j}^{t+1}} \\
& =\left[I-\frac{\Delta t}{2 S} v_{i, j} D^{*}+\frac{\Delta t}{2 S P r} B^{*}\right] \\
& {\left[I+\frac{\Delta t}{2 S} v_{i, j} D^{*}-\frac{\Delta t}{2 S P r} B^{*}\right]^{-1}} \\
& {\left[I-\frac{\Delta t}{2 S} u_{i, j} C^{*}+\frac{\Delta t}{2 S P r} A^{*}\right] T_{i, j}^{t}}
\end{aligned}
$$


From Eqns. (12), (13) and (14) we have:

$$
\begin{gathered}
T_{i, j}^{t+\frac{1}{2}}=E_{2} T_{i, j}^{t} \\
T_{i, j}^{t+1}=G_{2} T_{i, j}^{t+\frac{1}{2}} \\
T_{i, j}^{t+1}=G_{2} E_{2} T_{i, j}^{t}
\end{gathered}
$$

where,

$$
\begin{aligned}
& E_{2}=\left[I+\frac{\Delta t}{2 S} v_{i, j} D^{*}-\frac{\Delta t}{2 S P r} B^{*}\right]^{-1}\left[I-\frac{\Delta t}{2 S} u_{i, j} C^{*}+\frac{\Delta t}{2 S P r} A^{*}\right] \\
& G_{2}=\left[I+\frac{\Delta t}{2 S} u_{i, j} C^{*}-\frac{\Delta t}{2 S P r} A^{*}\right]^{-1}\left[I-\frac{\Delta t}{2 S} v_{i, j} D^{*}+\frac{\Delta t}{2 S P r} B^{*}\right]
\end{aligned}
$$

Writing the Eqns. (9) and (17) in matrix form we obtain:

$$
\left[\begin{array}{c}
\xi_{i, j}^{t+1} \\
T_{i, j}^{t+1}
\end{array}\right]=\left[\begin{array}{cc}
G_{1} E_{1} & G_{1} F_{1}+H_{1} \\
0 & G_{2} E_{2}
\end{array}\right]\left[\begin{array}{c}
\xi_{i, j}^{t} \\
T_{i, j}^{t}
\end{array}\right]
$$

For stability of the considered problem by matrix method, we must have:

$$
\left\|\left[\begin{array}{cc}
G_{1} E_{1} & G_{1} F_{1}+H_{1} \\
0 & G_{2} E_{2}
\end{array}\right]\right\| \leq 1
$$

which imply $\left\|G_{1} E_{1}\right\| \leq 1$ and $\left\|G_{2} E_{2}\right\| \leq 1$. For any matrix $M, \rho(M) \leq\|M\|$ all matrix norm $\|$.$\| . The equality can occur$ if we take norm to be spectral norm for the matrices, and it will be valid provided $M$ is diagonalizable with real eigenvalues. So in our case we have

$$
\rho\left(G_{i} E_{i}\right)=\left\|G_{i} E_{i}\right\| \leq\left\|G_{i}\right\|\left\|E_{i}\right\|=\rho\left(G_{i}\right) \rho\left(E_{i}\right) i=1,2
$$

Thus in order to achieve $\left\|G_{i} E_{i}\right\| \leq 1$, we will prove $\rho\left(G_{i}\right) \leq$ 1 and $\rho\left(E_{i}\right) \leq 1$ for $i=1$ and 2 . We will only prove that $\rho\left(E_{1}\right) \leq 1$ and the other case can be dealt similarly. Consider Eqns. (7) and (8) with $A^{*}=B^{*}$, and $C^{*}=D^{*}$. Note that this occurs for vorticity-transport equation due to uniform grid spacing in both directions [20]. The discretized Eq. (7) without temperature term can be expressed as:

$\left[-\frac{\Delta t}{4 \varepsilon \Delta y} v_{i, j}-\frac{\varepsilon \Lambda \Delta t}{2 \Delta y^{2}}\right] \xi_{i, j-1}^{t+\frac{1}{2}}+\left(1+\frac{\varepsilon \Lambda \Delta t}{\Delta y^{2}}\right) \xi_{i, j}^{t+\frac{1}{2}}+\left[\frac{\Delta t}{4 \varepsilon \Delta y} v_{i, j}-\frac{\varepsilon \Lambda \Delta t}{2 \Delta y^{2}}\right] \xi_{i, j+1}^{t+\frac{1}{2}}=$ $\left[\frac{\Delta t}{4 \varepsilon \Delta x} u_{i, j}+\frac{\varepsilon \Lambda \Delta t}{2 \Delta x^{2}}\right] \xi_{i, j-1}^{t}+\left(1-\frac{\varepsilon \Lambda \Delta t}{\Delta x^{2}}-\frac{\varepsilon \Delta t}{2 D a}\right) \xi_{i, j}^{t}+\left[-\frac{\Delta t}{4 \varepsilon \Delta x} u_{i, j}+\frac{\varepsilon \Lambda \Delta t}{2 \Delta x^{2}}\right] \xi_{i, j+1}^{t}$

Using the known boundary values and for a fixed $i$ in $x$ direction and varying the values for $j$ in $y$-direction, $j=2 \ldots n$ 1 , we get a system of equations expressed in matrix form as follows:

$$
\left[\begin{array}{ccccc}
a & b & & & \\
c & a & b & & \\
& \cdot & \cdot & \cdot & \\
& & c & a & b \\
& & & c & a
\end{array}\right]\left[\begin{array}{c}
\xi_{i, j_{2}}^{t+\frac{1}{2}} \\
\xi_{i, j_{3}}^{t+\frac{1}{2}} \\
\vdots \\
\xi_{i, j_{n-1}}^{t+\frac{1}{2}}
\end{array}\right]=\left[\begin{array}{ccccc}
a^{\prime} & b^{\prime} & & & \\
c^{\prime} & a^{\prime} & b^{\prime} & & \\
& \cdot & \cdot & \cdot & \\
& & c^{\prime} & a^{\prime} & b^{\prime} \\
& & & c^{\prime} & a^{\prime}
\end{array}\right]\left[\begin{array}{c}
\xi_{i, j_{2}}^{t} \\
\xi_{i, j_{3}}^{t} \\
\vdots \\
\xi_{i, j_{n-1}}^{t}
\end{array}\right]+R h_{j}
$$

where,

$$
\begin{aligned}
& a=\left[1+\frac{\varepsilon \Lambda \Delta t}{\Delta y^{2}}\right], \\
& b=\left[\frac{\Delta t}{4 \varepsilon \Delta y} v_{i, j}-\frac{\varepsilon \Lambda \Delta t}{2 \Delta y^{2}}\right], \\
& c=\left[-\frac{\Delta t}{4 \varepsilon \Delta y} v_{i, j}-\frac{\varepsilon \Lambda \Delta t}{2 \Delta y^{2}}\right] \\
& a^{\prime}=\left[1-\frac{\varepsilon \Lambda \Delta t}{\Delta x^{2}}-\frac{\varepsilon \Delta t}{2 D a}\right], \\
& b^{\prime}=\left[-\frac{\Delta t}{4 \varepsilon \Delta x} u_{i, j}+\frac{\varepsilon \Lambda \Delta t}{2 \Delta x^{2}}\right], \\
& c^{\prime}=\left[\frac{\Delta t}{4 \varepsilon \Delta x} u_{i, j}+\frac{\varepsilon \Lambda \Delta t}{2 \Delta x^{2}}\right]
\end{aligned}
$$

and $R h_{j}$ is the vector of known boundary values and zeros. Rewriting the above system:

$$
P_{1} \xi^{t+\frac{1}{2}}=Q_{1} \xi^{t}+R_{1}^{t+\frac{1}{2}}
$$

where, the matrices $P_{l}, Q_{1}$ are of order $n-2$ as shown above, $\xi^{t+\frac{1}{2}}$ and $\xi^{t}$ denotes the column vector with components $\xi_{i, j_{2}}{ }^{t+\frac{1}{2}}, \xi_{i, j_{3}}{ }^{t+\frac{1}{2}} \ldots \xi_{i, j_{n-1}}{ }^{t+\frac{1}{2}} \quad$ and $\quad \xi_{i, j_{2}}{ }^{t}, \xi_{i, j_{3}}{ }^{t} \ldots \xi_{i, j_{n-1}}{ }^{t}$ respectively, $R_{1}{ }^{t+\frac{1}{2}}$ is a column vector of boundary values and zeros of $\xi^{t+\frac{1}{2}}$. Since $P_{l}$ is a tri-diagonal matrix, the eigenvalues of matrix $P_{l}$ is given as [21]:

$$
\lambda_{s}=a+2 \sqrt{b c} \cos \left(\frac{s \pi}{n-1}\right), s=1,2, \ldots, n-2 .
$$

Using the values of $a, b, c$ from Eq. (18), the eigenvalues of matrix $P_{l}$ are given by

$\lambda_{p s}=\left(1+\frac{\varepsilon \Lambda \Delta t}{\Delta y^{2}}\right)+\frac{\varepsilon \Lambda \Delta t}{\Delta y^{2}}\left[\sqrt{1-\left(\frac{v_{i, j} \Delta y}{2 \varepsilon^{2} \Lambda}\right)^{2}}\right] \cos \left(\frac{s \pi}{n-1}\right), s=1,2, \ldots, n-2$.

Similarly, the eigenvalues of matrix $Q_{l}$ are given by

$\lambda_{q s}=\left(1-\frac{\varepsilon \Lambda \Delta t}{\Delta x^{2}}-\frac{\varepsilon \Delta t}{2 D a}\right)+\frac{\varepsilon \Lambda \Delta t}{\Delta x^{2}}\left[\sqrt{1-\left(\frac{u_{i, j} \Delta x}{2 \varepsilon^{2} \Lambda}\right)^{2}}\right] \cos \left(\frac{s \pi}{n-1}\right), s=1,2, \ldots, n-2$.

Hence, for stability we must have:

$$
\begin{aligned}
& \left\|P_{1}^{-1} Q_{1}\right\|_{2}=\rho\left(P_{1}^{-1} Q_{1}\right)=\max _{s}\left|\frac{\lambda_{q s}}{\lambda_{p s}}\right| \leq 1, \quad s=1,2, \ldots, n-2 . \\
& \left\|P_{1}^{-1} Q_{1}\right\|_{2}=\max _{s}\left|\frac{\left(1-\frac{\varepsilon \Lambda \Delta t}{\Delta x^{2}}-\frac{\varepsilon \Delta t}{2 D a}\right)+\frac{\varepsilon \Lambda \Delta t}{\Delta x^{2}}\left[\sqrt{1-\left(\frac{u_{i, j} \Delta x}{2 \varepsilon^{2} \Lambda}\right)^{2}}\right] \cos \left(\frac{s \pi}{n-1}\right)}{\left(1+\frac{\varepsilon \Lambda \Delta t}{\Delta y^{2}}\right)+\frac{\varepsilon \Lambda \Delta t}{\Delta y^{2}}\left[\sqrt{1-\left(\frac{v_{i, j} \Delta y}{2 \varepsilon^{2} \Lambda}\right)^{2}}\right] \cos \left(\frac{s \pi}{n-1}\right)}\right| \leq 1
\end{aligned}
$$

We have chosen,

$\Delta x=\Delta y, r=\frac{\varepsilon \Lambda \Delta t}{\Delta x^{2}}=\frac{\varepsilon \Lambda \Delta t}{\Delta y^{2}}, r^{\prime}=\frac{\varepsilon \Delta t}{2 D a}, M=\frac{u_{i, j} \Delta x}{2 \varepsilon^{2} \Lambda}$ and $M^{\prime}=\frac{v_{i, j} \Delta y}{2 \varepsilon^{2} \Lambda}$ 
Then the Eq. (20) can be expressed in the form:

$$
\left\|P_{1}^{-1} Q_{1}\right\|_{2}=\max _{s}\left|\frac{1-r-r^{\prime}+r \sqrt{1-M^{2}} \cos \left(\frac{s \pi}{n-1}\right)}{1+r+r \sqrt{1-M^{\prime 2}} \cos \left(\frac{s \pi}{n-1}\right)}\right| \leq 1, \quad s=1,2, \ldots, n-2
$$

Since $0 \leq u_{i, j}, v_{i, j} \leq 1, \Delta x^{2} \approx 10^{-6}, \varepsilon=0.2$, we have $|M| \leq 1,\left|M^{\prime}\right| \leq 1$ or $\left|\frac{u_{i, j} \Delta x}{2 \varepsilon^{2} \Lambda}\right| \leq 1,\left|\frac{v_{i, j} \Delta y}{2 \varepsilon^{2} \Lambda}\right| \leq 1$. So we get $\left|u_{i, j} \Delta x\right| \leq 2 \varepsilon^{2} \Lambda$ and $\left|v_{i, j} \Delta y\right| \leq 2 \varepsilon^{2} \Lambda$. Now assuming:

$$
\begin{gathered}
f=\frac{1-r-r^{\prime}+r \sqrt{1-M^{2}} \cos \left(\frac{s \pi}{n-1}\right)}{1+r+r \sqrt{1-M^{\prime 2}} \cos \left(\frac{s \pi}{n-1}\right)}, \text { we see that } \\
\frac{d f}{d s}=0 \quad \text { gives } s=n-1,
\end{gathered}
$$

For this value of $\mathrm{s},\left\|P_{1}^{-1} Q_{1}\right\|_{2}$ has maximum value, therefore Eq. (21) reduces to:

$$
\left|\frac{1-r-r^{\prime}-r \sqrt{1-M^{2}}}{1+r-r \sqrt{1-M^{\prime 2}}}\right| \leq 1
$$

Left side inequality implies,

$$
-1-r+r \sqrt{1-M^{\prime 2}} \leq 1-r-r^{\prime}-r \sqrt{1-M^{2}}
$$

On simplifying

$$
r<\frac{2}{\left[\sqrt{1-M^{2}}+\sqrt{1-M^{2}}\right]}
$$

Letting $M, M^{\prime} \rightarrow 0, r<1$. Also,

$$
0 \leq \frac{\varepsilon \Lambda \Delta t}{\Delta x^{2}}=\mathrm{r}<1 \text {, i.e. } \Delta t<\frac{\Delta x^{2}}{\varepsilon \Lambda}
$$

Now consider the case $\rho\left(E_{2}\right) \leq 1$ and the case $\rho\left(G_{2}\right) \leq$ 1 follows on similar lines. Now, $E_{2}$ is equivalent to matrix form as described below:

$$
\left[\begin{array}{ccccc}
d & e & & & \\
f & d & e & & \\
& \cdot & \cdot & \cdot & \\
& & f & d & e \\
& & & f & d
\end{array}\right]\left[\begin{array}{c}
T_{i, j_{2}}^{t+\frac{1}{2}} \\
T_{i, j_{3}}^{t+\frac{1}{2}} \\
\vdots \\
T_{i, j_{n-1}}^{t+\frac{1}{2}}
\end{array}\right]=\left[\begin{array}{ccccc}
d^{\prime} & e^{\prime} & & & \\
f^{\prime} & d^{\prime} & e^{\prime} & & \\
& \cdot & \cdot & \cdot & \\
& & f^{\prime} & d^{\prime} & e^{\prime} \\
& & & f^{\prime} & d^{\prime}
\end{array}\right]\left[\begin{array}{c}
T_{i, j_{2}}^{t} \\
T_{i, j_{3}}^{t} \\
\vdots \\
T_{i, j_{n-1}}^{t}
\end{array}\right]+R h^{\prime}{ }_{j}
$$

where,

$$
\begin{aligned}
& d=\left[1+\frac{\Delta t}{\operatorname{SPr} \Delta y^{2}}\right], e=\left[\frac{\Delta t}{4 S \Delta y} v_{i, j}-\frac{\Delta t}{2 S \operatorname{Pr} \Delta y^{2}}\right], f=\left[-\frac{\Delta t}{4 S \Delta y} v_{i, j}-\frac{\Delta t}{2 S \operatorname{Pr} \Delta y^{2}}\right] \\
& d^{\prime}=\left[1-\frac{\Delta t}{S \operatorname{Pr} \Delta x^{2}}\right], e^{\prime}=\left[-\frac{\Delta t}{4 S \Delta x} u_{i, j}+\frac{\Delta t}{2 S \operatorname{Pr} \Delta x^{2}}\right], f^{\prime}=\left[\frac{\Delta t}{4 S \Delta x} u_{i, j}+\frac{\Delta t}{2 S \operatorname{Pr} \Delta x^{2}}\right]
\end{aligned}
$$

and $R h_{j}^{\prime}$ is the vector of known boundary values and zeros. Rewriting,

$$
P_{2} T^{t+\frac{1}{2}}=Q_{2} T^{t}+R_{2}^{t+\frac{1}{2}}
$$

where, the matrices $P_{2}, Q_{2}$ are of order $n-2$ as shown above, $T^{t+\frac{1}{2}}$ and $T^{t}$ denotes the column vector with components $T_{i, j_{2}}{ }^{t+\frac{1}{2}}, T_{i, j_{3}}{ }^{t+\frac{1}{2}} \ldots T_{i, j_{n-1}}{ }^{t+\frac{1}{2}} \quad$ and $\quad T_{i, j_{2}}{ }^{t}, T_{i, j_{3}}{ }^{t} \ldots T_{i, j_{n-1}}{ }^{t}$ respectively, $R_{2}{ }^{t+\frac{1}{2}}$ is a column vector of boundary values and zeros of $T^{t+\frac{1}{2}}$. Now, proceeding as for the above case, we get:

$$
\Delta t<S \operatorname{Pr} \Delta x^{2}
$$

Thus, we have proved that:

$$
\begin{gathered}
\left|u_{i, j} \Delta x\right| \leq 2 \varepsilon^{2} \Lambda,\left|v_{i, j} \Delta y\right| \leq 2 \varepsilon^{2} \Lambda, \Delta t<\frac{\Delta x^{2}}{\varepsilon \Lambda}, \\
\left|u_{i, j} \Delta x \operatorname{Pr}\right| \leq 2,\left|v_{i, j} \Delta y \operatorname{Pr}\right| \leq 2, \Delta t<\operatorname{SPr} \Delta x^{2} .
\end{gathered}
$$

Thus the applied numerical scheme for the considered problem is unconditionally stable. The considered scheme is also consistent as local truncation error tends to zero as the step lengths in all direction tends to zeros. Hence the considered scheme is convergent by Lax's equivalence theorem [21].

\section{NUMERICAL COMPUTATIONS}

The numerical solution of the unknown flow variables $\psi, \xi, T$ for the considered problem has been calculated with the help of MATLAB. We have chosen the relevant parameters in the governing equations like Prandtl number $(\mathrm{Pr})$ and Darcy number $(D a)$, porosity $(\varepsilon)$, Grashof number $(G r)$ compatible with the considered problem.

\subsection{Algorithms for stream function-vorticity formulation}

(i) Set initial conditions at time $t=0$ for $\psi, \xi, T$.

(ii) Calculate $\xi_{i, j}$ at time level $t+\Delta t$ for each interior grid point from (13) and (17).

(iii) Obtain $\psi_{i, j}$ at all points by solving Eq. (3) using new $\xi$ values at interior points.

(iv) Update velocities by calculating $u=\frac{\partial \psi}{\partial y}, v=-\frac{\partial \psi}{\partial x}$.

(v) Calculate $T_{i, j}$ at time level $t+\Delta t$ for each interior grid point from (14) and (18) using $u$ and $v$ values at interior points.

(vi) Update $\xi_{i, j}$ at the boundaries using $\psi$ and $\xi$ values at internal points.

(vii) Return to Step (ii) if the solution is not converged.

\section{RESULTS AND DISCUSSION}

This section discusses the numerical results of streamline contours, isotherm contours, and the average Nusselt number at the left and right walls of the square enclosure. The results are analyzed for different values of Darcy number $(D a=0.01$, $0.001)$, viscosity ratio $(\Lambda=1,3)$, Grashof number $(G r=$ $50,000)$, porosity $(\varepsilon=0.2)$, and Prandtl number $(P r=7)$ for two different cases as mentioned above. The numerical computations are carried out in a transient form at different time levels $t=0.01,0.1,0.5,1,10$ and many more We have observed that the numerical results attain their steady-state solutions for Darcy number $D a=0.001$ and the viscosity ratios $(\Lambda=1,3)$ at time level $t=1.5$. In contrast, it has oscillating behavior for Darcy number $D a=0.01$ and both the viscosity ratios $(\Lambda=1,3)$ after a specific time period. 


\subsection{Case: 1}

Figures 2 to 5 present the streamline contours for a parallel sided lid-driven cavity problem with different values of Darcy number $(D a=0.01,0.001)$ and viscosity ratios $(\Lambda=1,3)$ at various time levels. The streamline contours have different behavior for distinct viscosity ratios at $D a=0.01$. Figure 2 depicts the streamline contours for Darcy number $D a=0.01$ and viscosity ratio $(\Lambda=1)$ at distinct time levels. The streamline contours attain an elliptic shape near the horizontal walls of the cavity at time level $t=0.01$. The two small circular type contours are generated near the left and right wall of the cavity, inside a single contour of absolute value 0.1 at $t=0.1$. The four contours are formed about the horizontal and vertical line through the mid of the cavity at $t=0.5$. As time increases from $t=0.5$ to 1 , the two small contours on the diagonal corner along with a large circular contour with centre at $(0.5,0.5)$ are developed. This large circular contour moves in an anticlockwise direction. With an increment of time $t=1$ to 2.6 , the weaker top-diagonal contour starts expanding (with a clockwise rotation) and shifting the large anti-clockwise rotating contour towards the right vertical wall. And finally, the clockwise rotating contour occupies the whole cavity with two weaker contours in the anti-diagonal corner of the cavity at time level $t=2.7$.

The weaker contour in the left-bottom corner of the cavity starts expanding with an anti-clockwise rotation and vanishing the larger clockwise rotating shape with an increment of time $t=2.7$ to 5.2. The streamline contours attain the same position
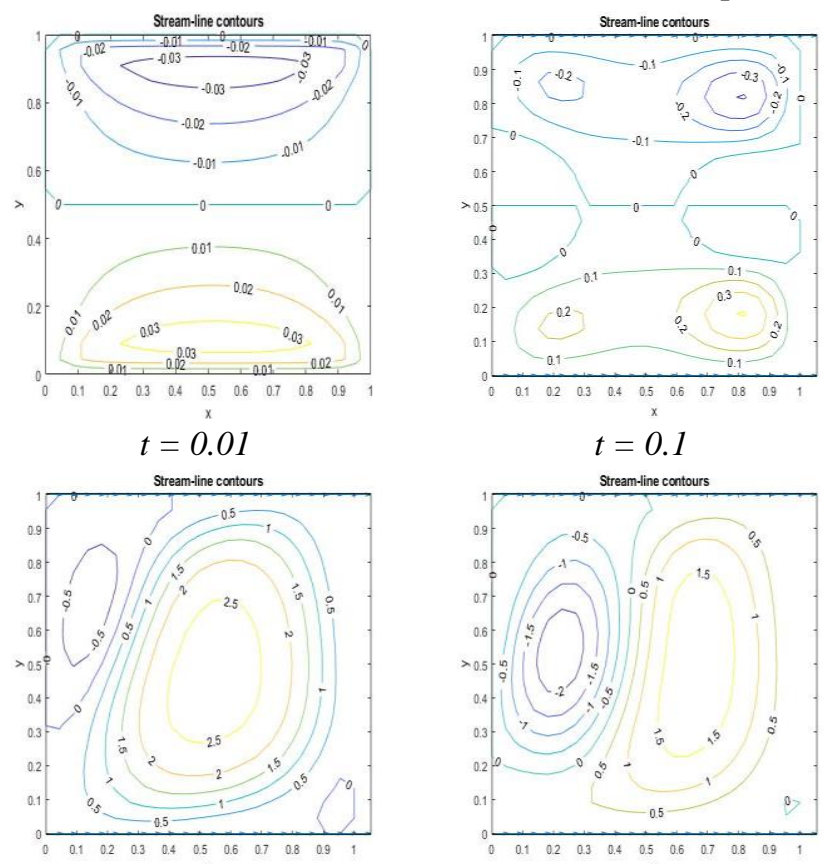

$t=2.5$

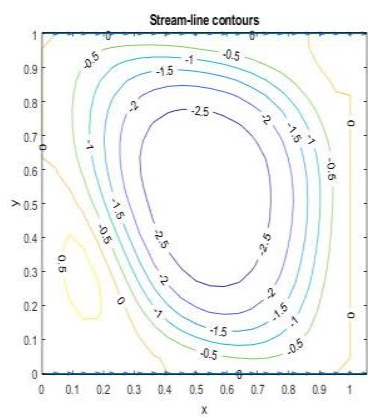

$t=4.5$

Figure 2. Streamline contours for two-sided lid-driven square porous $t=4$

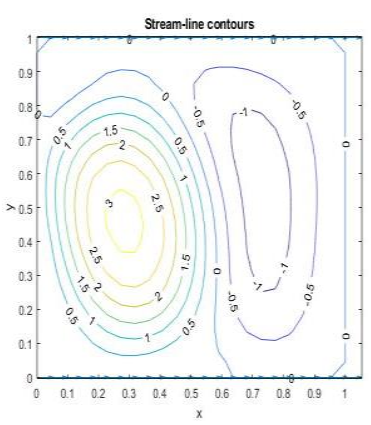

for two as it was at the time level of $t=1$. This occupying and vanishing the clockwise or anti-clockwise rotating shapes continue as time increases due to the oscillating behavior in the average Nusselt number at $D a=0.01$ (see Figure 10).

Figure 3 illustrates the streamlined contours for $D a=0.01$ and viscosity ratio $(\Lambda=3)$ at different time levels. The streamline contours of viscosity ratio $(\Lambda=3)$ are similar to $\Lambda$ $=1$ at time $t=0.01$, but the intensity of the streamline contours are higher than that of $\Lambda=1$. At $t=0.1$, the innermost streamline contours regarding the mid-horizontal line through the geometric centre of the cavity look like a human foot as it has a depression. The streamline contours above the geometric centre of the cavity have a clockwise rotation. In contrast, it has an anti-clockwise rotation in the bottom half of the cavity. It attains a nano-car structure as time increases 0.5 to 1 . This behavior retains till time level $t=7.8$, and then it starts changing its behavior as time increases. As time increases $t=$ 10 to 11.2 , the clockwise rotating elliptic streamline contours dominate the anti-clockwise rotating contours present in the lower half of the cavity. The streamline contours in the lower half of the domain start expanding and shifts the clockwise streamlined contours in the top left corner of the cavity as time increases from 11.33 to 11.37 . With an increment in time $t=$ 11.37 to 11.48 , the clockwise rotating streamlined contours start dominating the anti-clockwise one in the lower half of the cavity. This process continues as time increases due to the periodicity of the average Nusselt number for $D a=0.01$ and $\Lambda$ $=3($ see Figure 10).
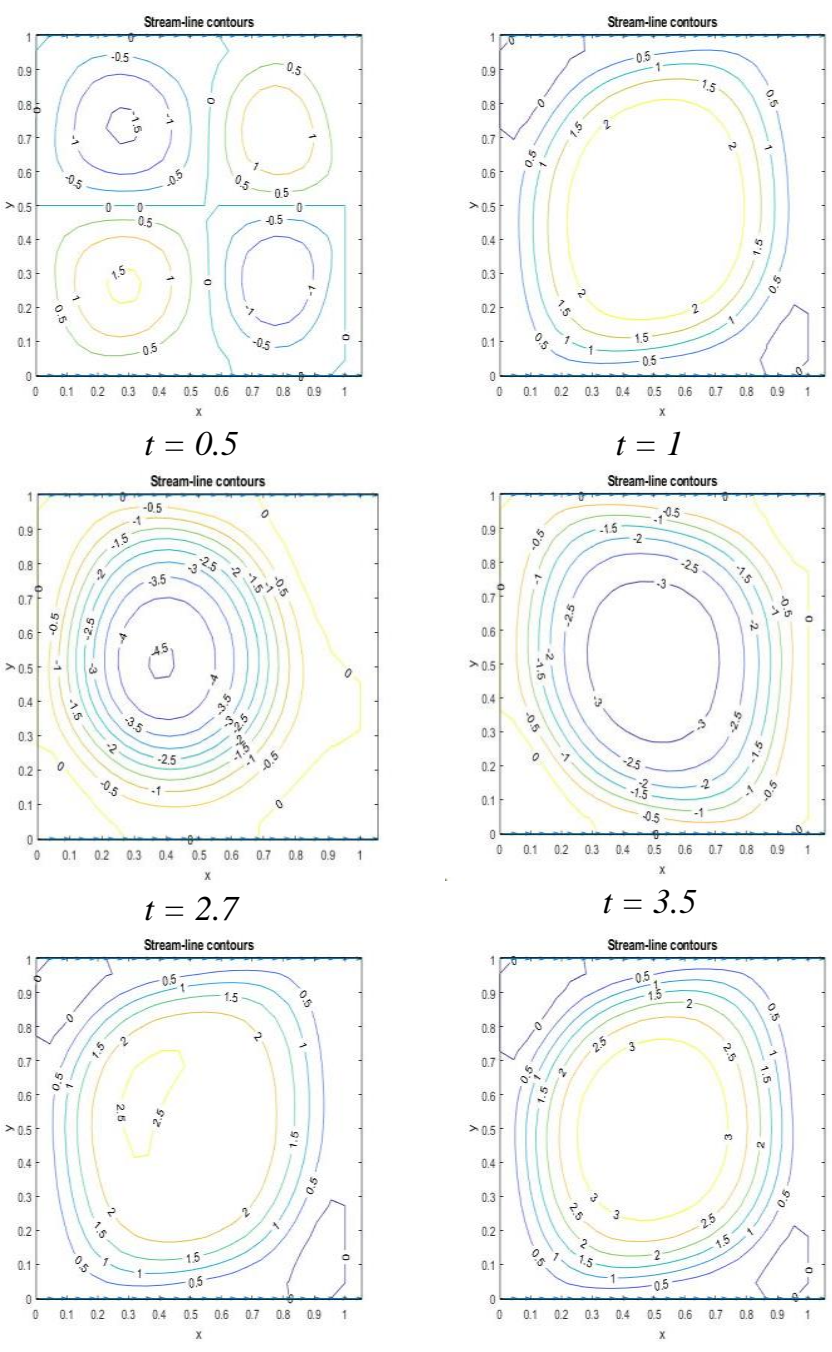

$$
t=5.2
$$



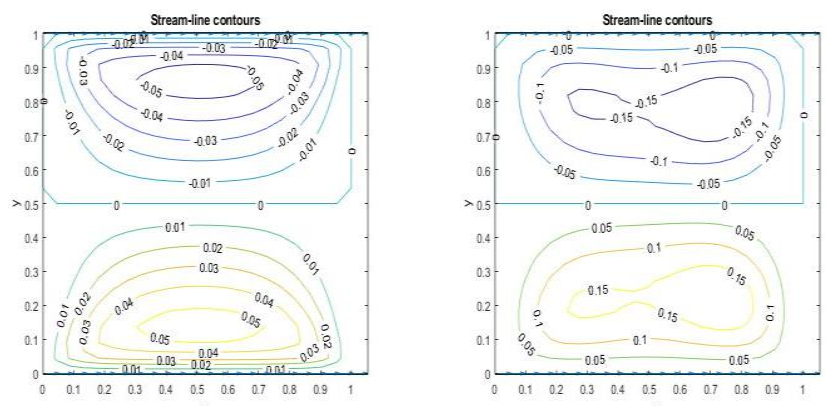

$t=0.01$

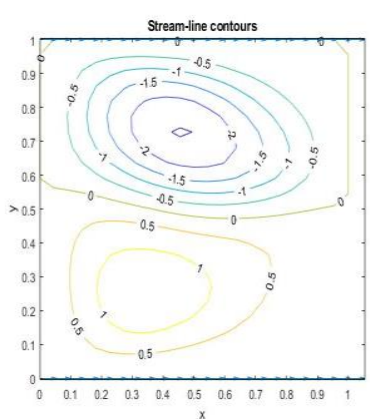

$t=10$

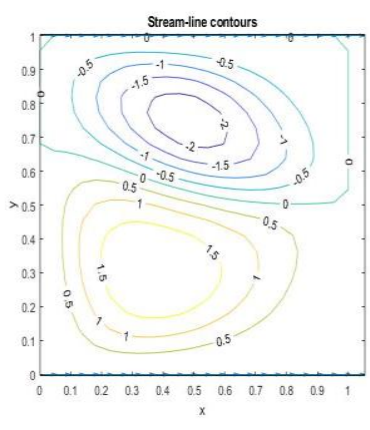

$t=11.48$

$t=0.1$

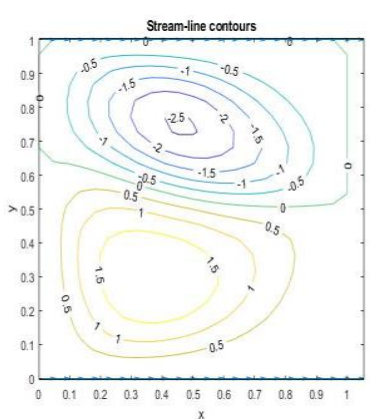

$t=11.2$

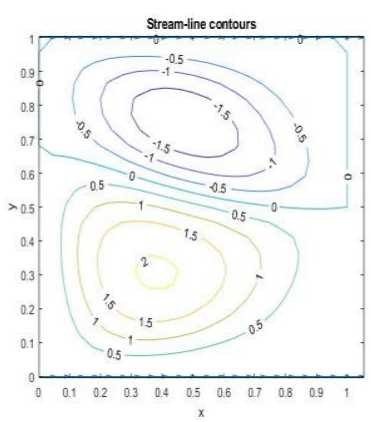

$t=11.50$

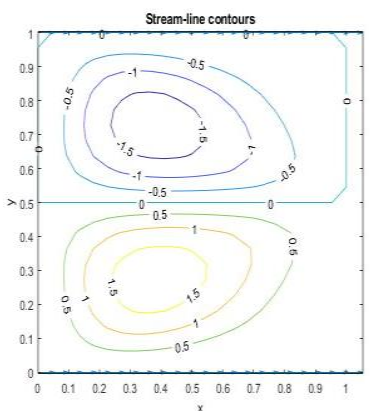

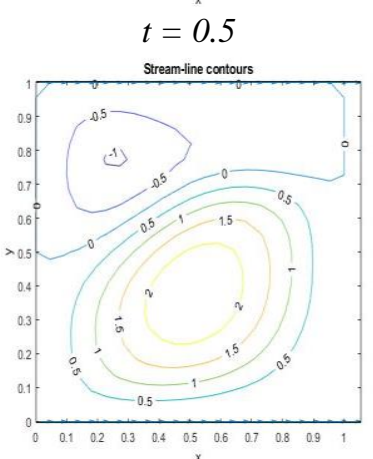

$t=11.33$

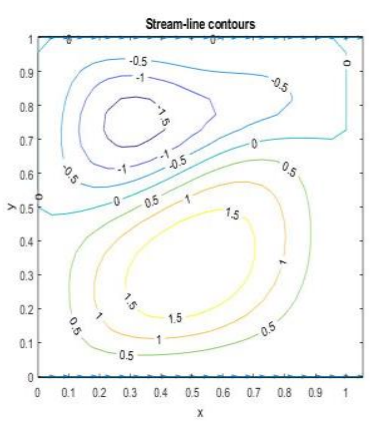

$t=11.70$

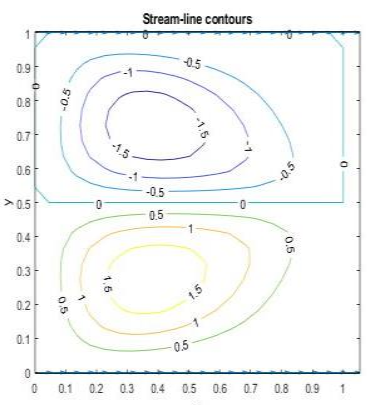

$t=1$

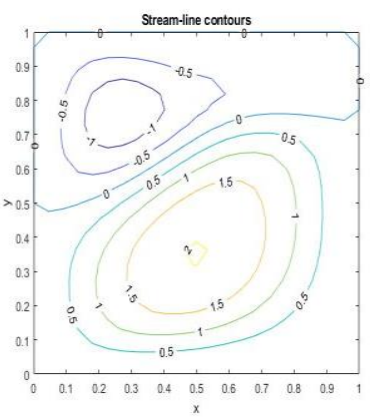

$t=11.37$

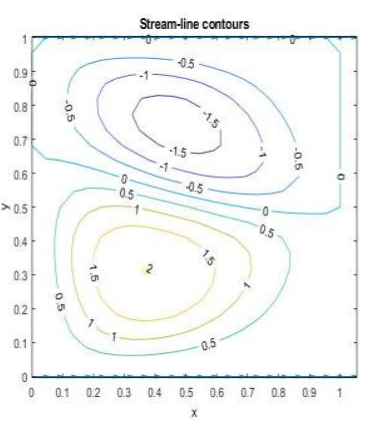

$t=11.8$

Figure 3. Streamline contours for two-sided lid-driven square porous cavity with $D a=0.01$ and $\Lambda=3$

Figures 4 and 5 show the streamline contours for $D a=0.001$ with viscosity ratios $\Lambda=1$ and $\Lambda=3$ respectively. The streamline contours for $\Lambda=1$ and $\Lambda=3$ are having a similar behavior at a particular time level $t=0.01,0.1,0.5,1,10$. The streamlined contours about the horizontal centerline of the cavity are elliptic. They have a bend towards the mid of the right vertical wall of the domain for both the viscosity ratio $\Lambda$ $=1,3$. The difference is in the magnitude of the stream function $\psi$. The absolute value of stream function $\psi$ decreased with an increase of viscosity ratio $\Lambda=1$ to 3 and a decrease of Darcy number from $D a=0.01$ to 0.001 .

The isotherm contours for different values of Darcy number $(D a=0.01,0.001)$, viscosity ratio $(\Lambda=1,3)$ at different time levels are presented in Figures 6 to 9. The isotherm contours shift towards the cavity's midpoint with an increment of time $t$ $=0.01$ to 0.1 . These contours settle near the domain's boundary at time $t=1$. As time increases from 1 to 2.6 , the isotherm contours occupy almost the whole cavity except the middle part of the lower half of the domain. The left-half region of the cavity contains cold profiles, while the other half includes the hot one. With an increment of time from 2.6 to 4.5 , the cold isotherm contours start occupying the whole cavity by pushing the hot isotherms towards the right wall. The contours occupy a $\psi$ type structure and leave the top middle region of the domain at $t=4.7$. Further, as time increases 4.7 to 5.2, isotherm contours attain the same structure as a case of time $t$ $=1$. Now, this process continues again and again due to the periodicity of the average Nusselt number.

Figure 7 depicts the isotherm contours for $D a=0.01$ and $\Lambda$ $=3$ at different time levels. The isotherm contours are symmetric about the horizontal line in the mid of the cavity at $t=0.5$. The temperature profiles shifts from the hot (right) wall to the cold (left) wall in an electric bulb-like structure. The cold isotherm from the left vertical wall moves along the top and bottom's mid-wall. While the hot isotherm contours move towards the left wall from the middle of the right wall at $t=$ 0.5 and 1 . This position shifts upward along the right boundary as time increases from 1 to 11.20 . Further, it tends antidiagonally as time rises from 11.20 to 11.37 . These contours start changing their position to the original one (at $t=1$ ), and then it oscillates between two shapes (that occurred at a time level 1 and 11.37) as time increases.

Figures 8 and 9 show the isotherm contours for $D a=0.001$ with viscosity ratio $\Lambda=1$ and 3 at distinct time levels. The isotherm contours behave like that of $D a=0.01, \Lambda=3$ till time $t=1$ and then attain its steady state. Figures 8 and 9 depict that the cold isotherm contours occupy the region near the left, top, and bottom wall of the cavity from $t=0.5$ onwards for both viscosity ratios 1 and $3, D a=0.001$. In contrast, the hot isotherm contours occupy the area near the right boundary and the middle part of the domain as a Conical Flask placed vertically at time level 0.5 and then attain a test-tube structure.

Figures 10 and 11 present the average Nusselt number along the left and right wall of the cavity for different values of 
Darcy number $(D a=0.01,0.001)$ and viscosity ratio $(\Lambda=1,3)$. Figure 10 illustrates the average Nusselt number at both left and right wall oscillate periodically after $t=2$ with a period of 2.06 for the viscosity ratio $\Lambda=1$ and $D a=0.01$. While for $\Lambda=$ 3 , the average Nusselt number at both side walls first attains a steady-state (at approximately $t=1$ ) and then starts oscillating with a minor frequency till 11.206. This frequency enlarges after 11.206 and attains a steady oscillating behavior with a period of 0.29 . Figure 11 shows that the average Nusselt number at both sides has the same behavior for both the viscosity ratio $\Lambda=1$ and 3 , at $D a=0.001$. The average Nusselt number at the left (or right) wall first decrease, then increases, again decreases, and settles steady-state solution at $t=1.25$. The numerical simulations were carried out till time level 40 and found it attains steady-state solutions after $t=1.25$.
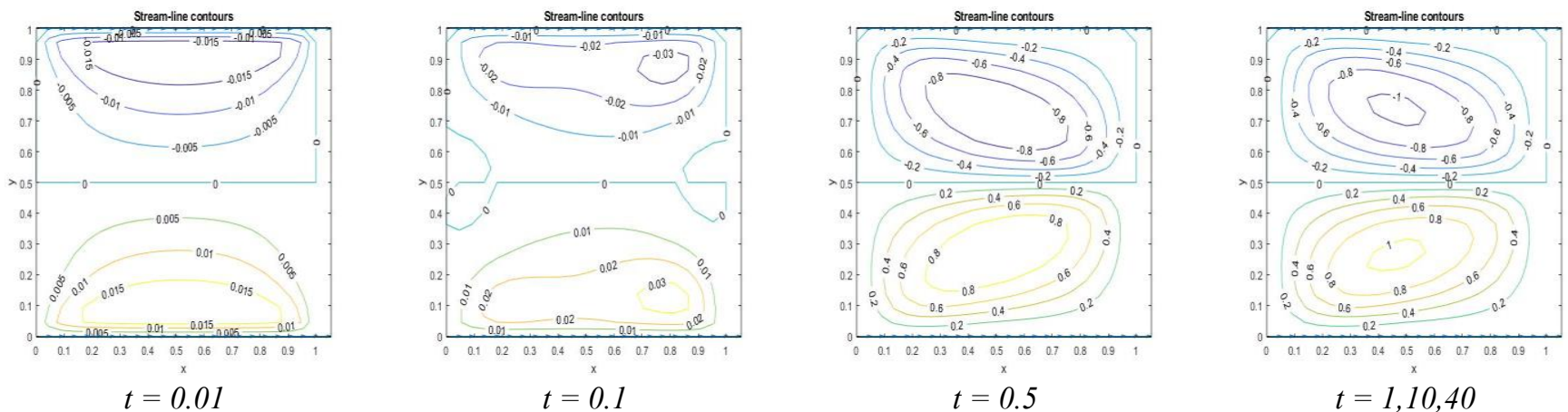

Figure 4. Streamline contours for two-sided lid-driven square porous cavity with $D a=0.001$ and $\Lambda=1$
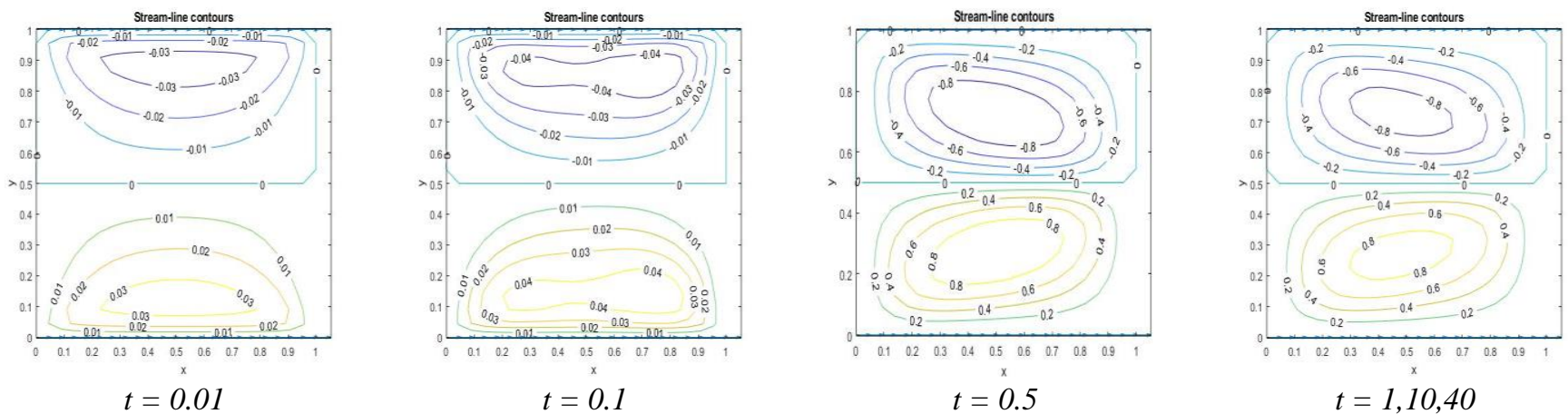

Figure 5. Streamline contours for two-sided lid-driven square porous cavity with $D a=0.001$ and $\Lambda=3$
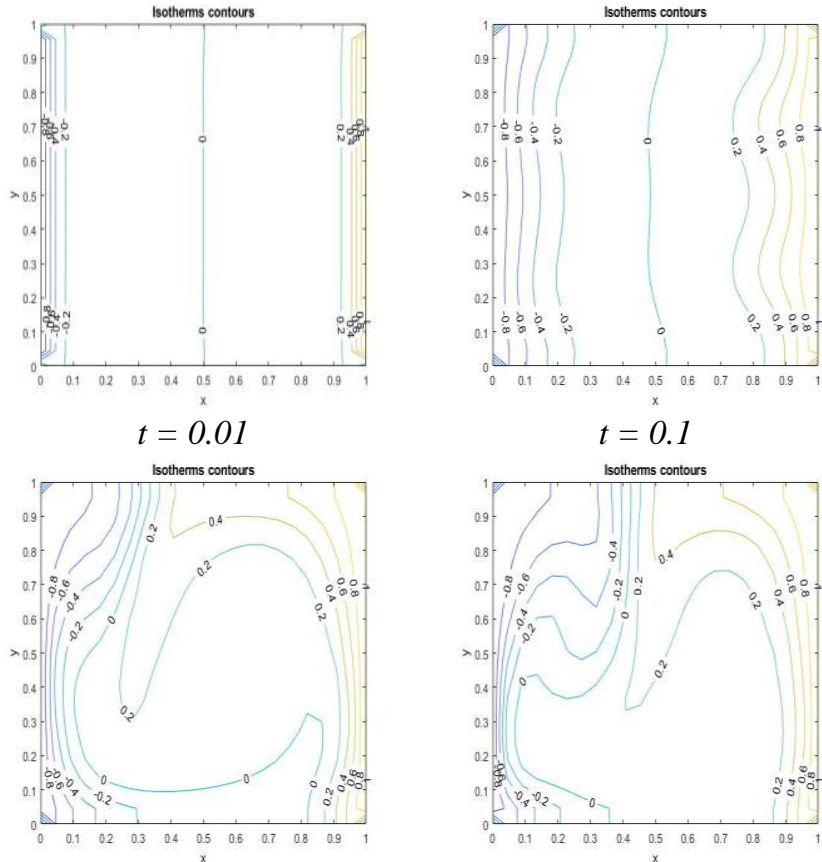

$t=2.5$

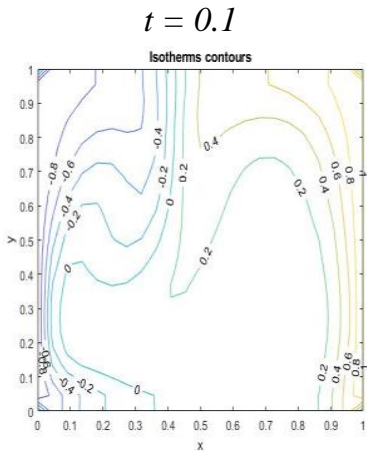

$t=2.6$
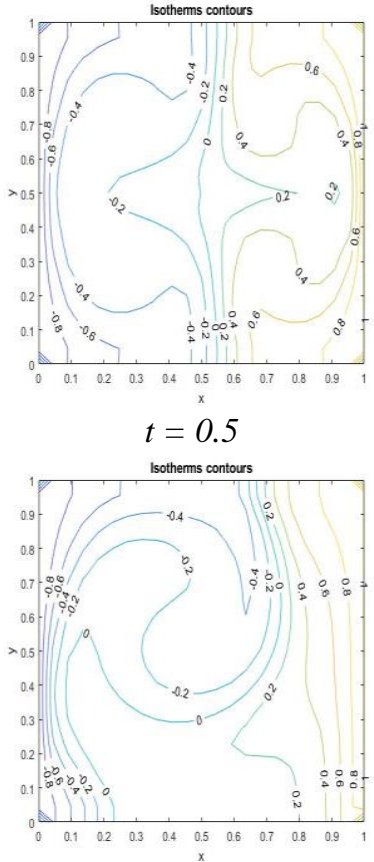

$t=2.7$
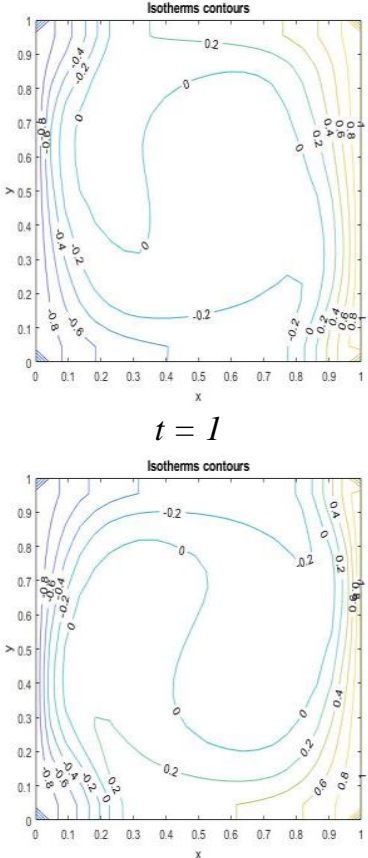

$t=3.5$ 


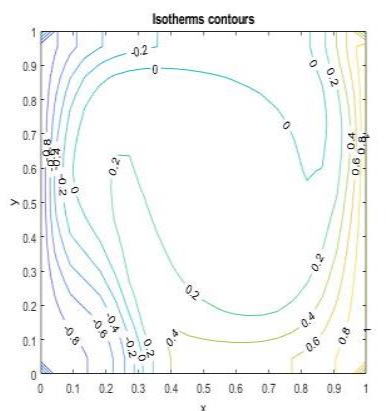

$t=4.5$

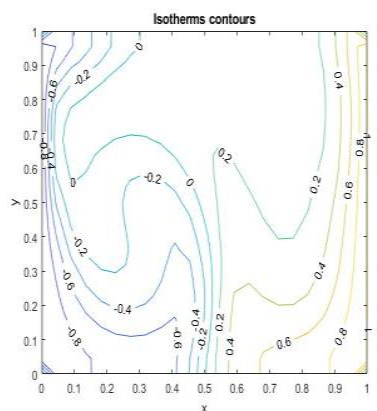

$t=4.7$

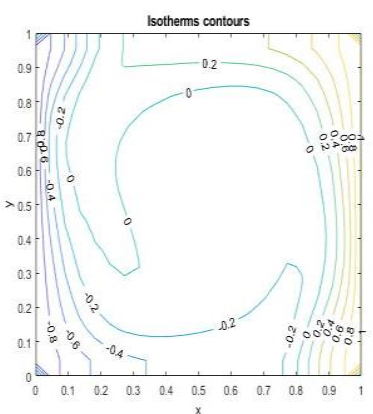

$t=4.9$

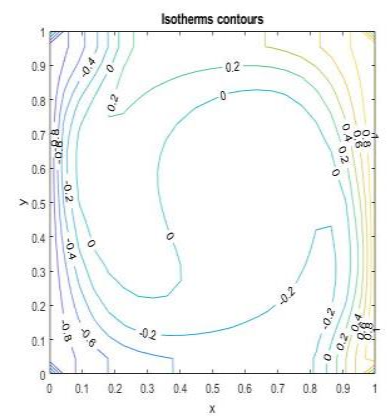

$t=5.2$

Figure 6. Isotherms contours for two-sided lid-driven square porous cavity with $D a=0.01$ and $\Lambda=1$
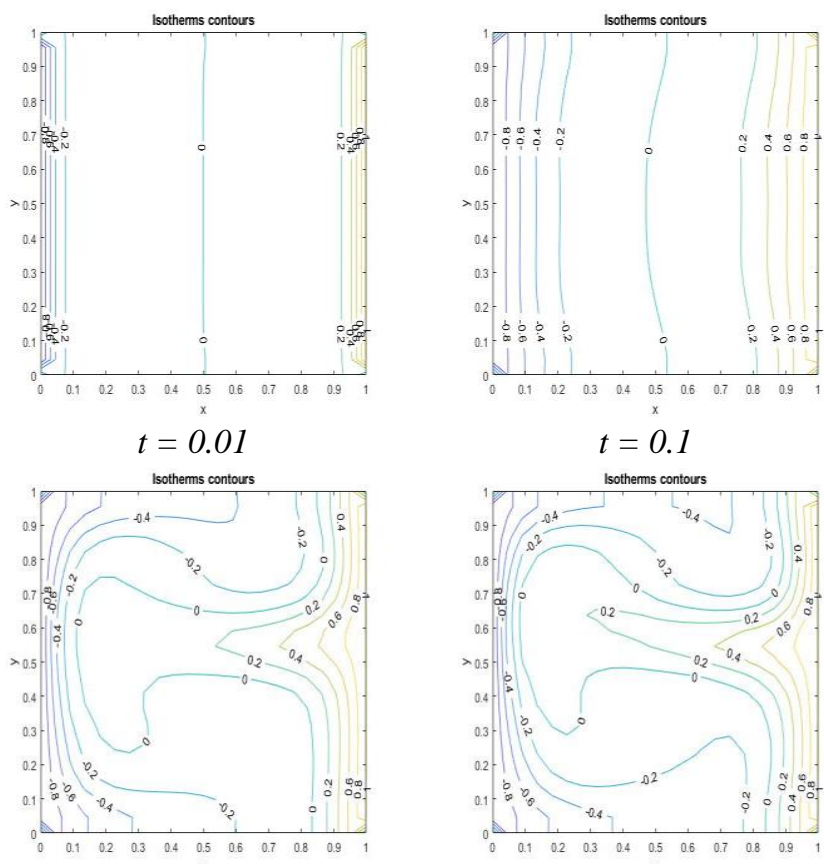

$$
t=10
$$

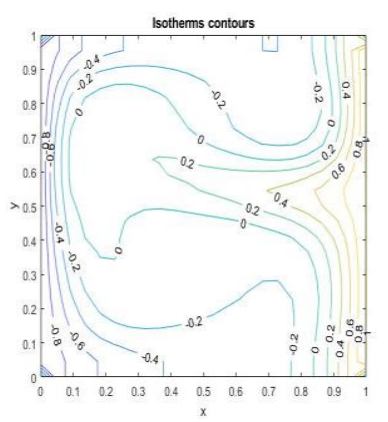

$t=11.48$

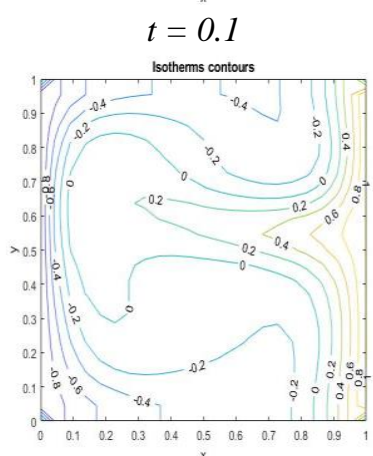

$$
t=11.2
$$

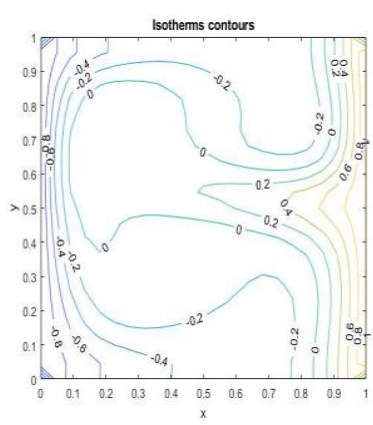

$t=11.50$

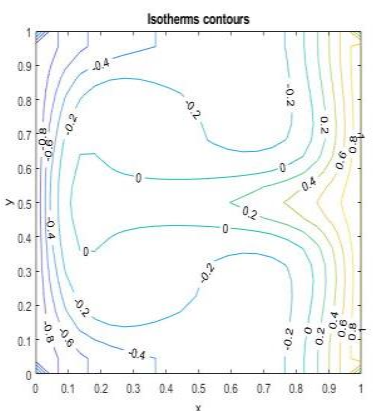

$t=0.5$

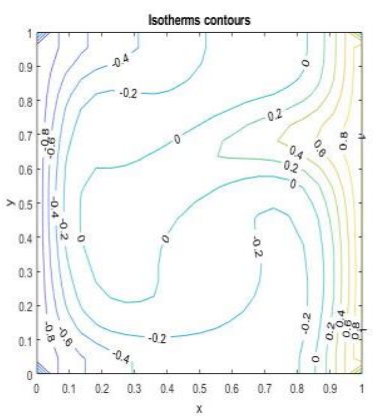

$t=11.33$

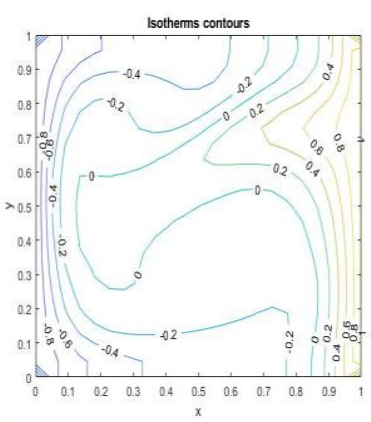

$t=11.70$

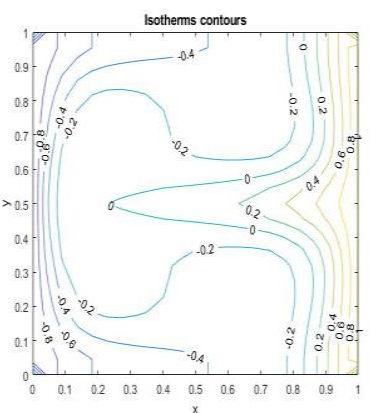

$t=1$

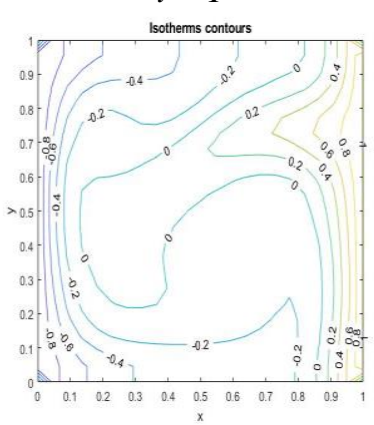

$$
t=11.37
$$

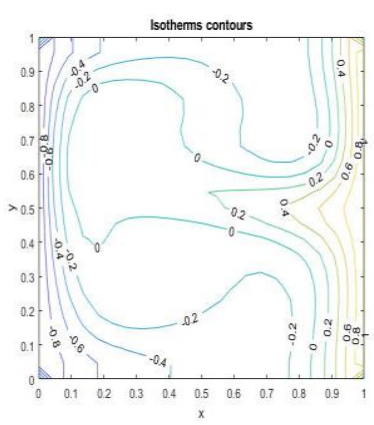

$t=11.80$

Figure 7. Isotherms contours for two-sided lid-driven square porous cavity with $D a=0.01$ and $\Lambda=3$
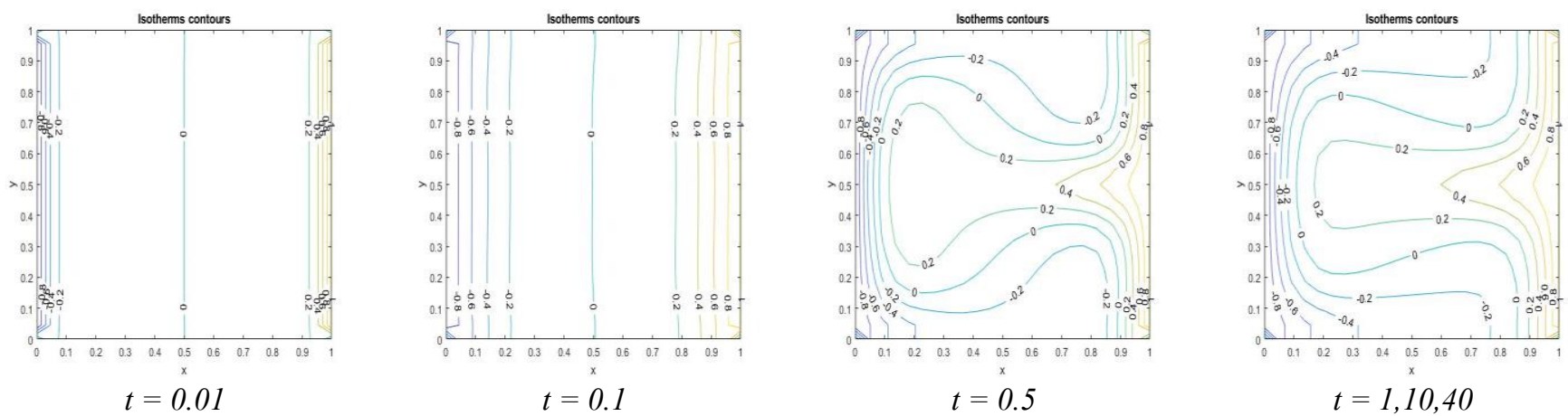

Figure 8. Isotherms contours for two-sided lid-driven square porous cavity with $D a=0.001, \Lambda=1$ 


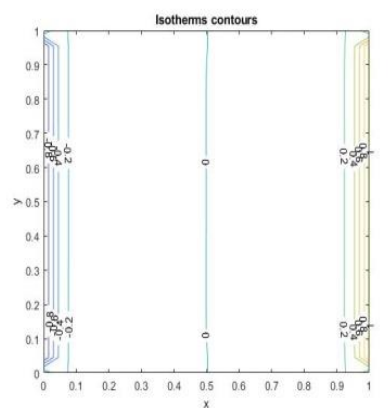

$t=0.01$

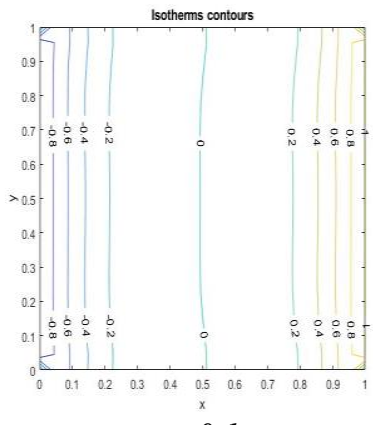

$t=0.1$
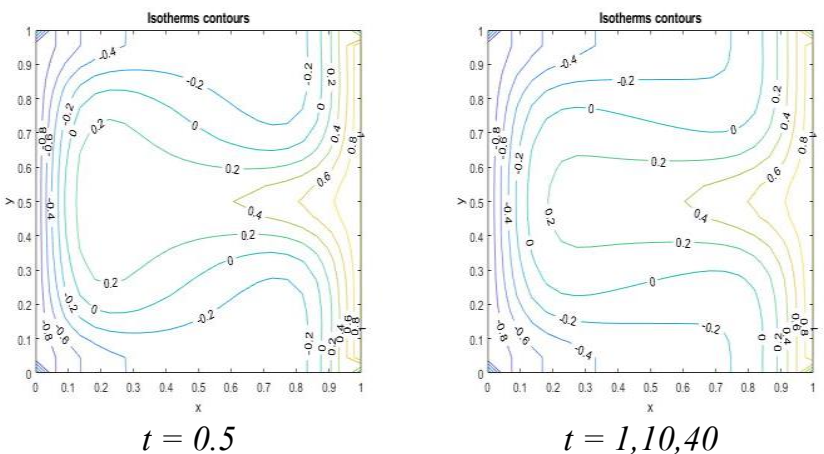

$t=1,10,40$

Figure 9. Isotherms contours for two-sided lid-driven square porous cavity with $D a=0.001, \Lambda=3$

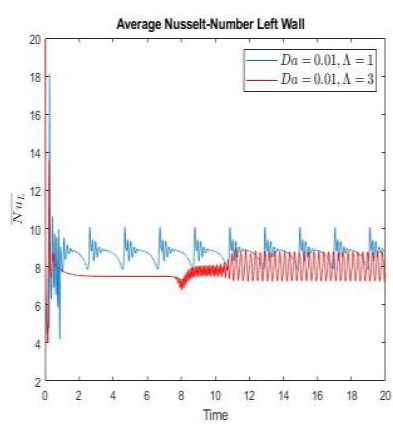

(a) Left Wall

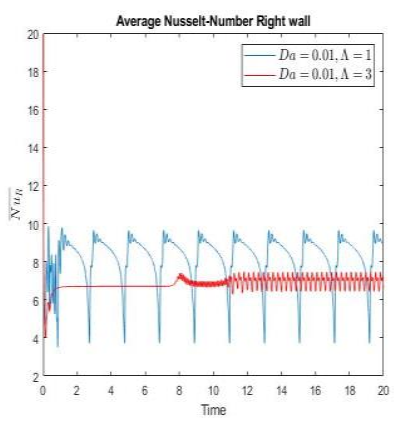

(b) Right Wall

Figure 10. Average Nusselt-Number for a two-sided liddriven square porous cavity with $D a=0.01, \Lambda=1$ and 3 , at the left and right wall

\subsection{Case: 2}

In this subsection, we will discuss the numerical results for Case 2, in which all the four walls of the cavity are kept moving. The top, bottom, and left, the right walls move with a uniform velocity from left to right, right to the left and top to down, down to the top, respectively. The top and bottom walls are maintained at constant temperature $T=1$ and -1 , respectively. The heat flux is provided at the left and right walls of the cavity.

The streamline contours for Darcy number $D a=0.01,0.001$ and viscosity ratio $\Lambda=1,3$, are depicted in Figures 12 and 13 at different time levels. The two streamline contours are formed one above and the other below the geometric centre of the cavity at $t=0.01$. These contours reduce to a single circular contour with some inclination towards the diagonal of the cavity at $t=0.1$. The two small contours inside large contours are generated as time increases from $t=0.1$ to 0.5 . These contours reduce their sizes with an increment of time and attain a steady-state at $t=1$ onwards. The inclination of the streamline contours towards the diagonal is greater for $\Lambda=1$ in comparison to $\Lambda=3$.

Figures 14 and 15 show two streamline contours are generated about the geometric center of the square cavity at $t$ $=0.01$. These two contours take a peanut-like shape with bends diagonally at $t=0.1$. The streamline contours are arranged almost perpendicular to the horizontal axis as time increased from $t=0.1$ to 0.5 and attained steady-state. The stream function $\psi$ decreases with increasing viscosity ratio $\Lambda$ $=1$ to 3 for Darcy number $D a=0.01$ and 0.001 . The stream function $\psi$ decreases with decreasing Darcy number from $D a$ $=0.01$ to 0.001 at a particular viscosity ratio.

Figures 16 to 19 present the isotherm contours for different values of Darcy number $(D a=0.01,0.001)$ and viscosity ratios $\Lambda=1$ and $\Lambda=3$ at different time level $t=0.01,0.1,0.5,1$ and

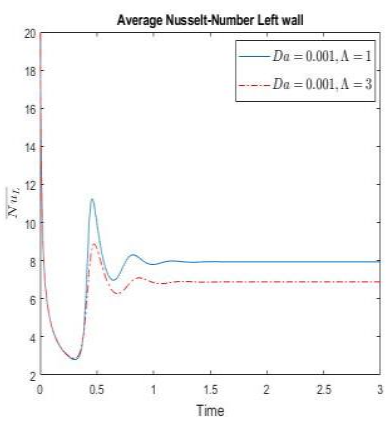

(a) Left Wall

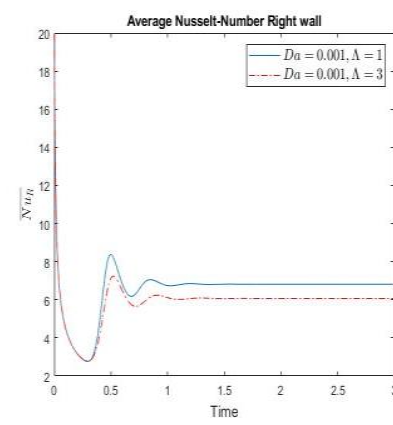

(b) Right Wall
Figure 11. Average Nusselt-Number for a two-sided liddriven square porous cavity with $D a=0.001, \Lambda=1$ and 3 , at left and right wall

10. Figures 16 and 17 show that the isotherm contours are straight lines near the top and bottom walls of the cavity at $t=$ 0.01 . The isotherm contours of the top wall shift towards the cavity's left wall at $t=0.1$ while contours of the bottom wall shift towards the right wall. The maximal temperature between the top and bottom walls increases from 0 to 0.2 as time changes from 0.01 to 0.1 . In the middle of the cavity, isotherm contours bend sharply for viscosity ratio $\Lambda=1$ in comparison to $\Lambda=3$. As time changes from 0.1 to 0.5 , isotherm contours from the bottom wall increases then decreases and again increases to approach the top wall for both viscosity ratio $(\Lambda=$ $1,3)$. The effect of viscosity ratio is negligible after $t=0.5$ onwards. The isotherm contours attain their steady-state solution after $t=0.5$.

Figures 16 to 19 illustrate that the isotherm contours behave almost the same for Darcy numbers $D a=0.01$ and 0.001 . The variation in contours becomes negligible for $\Lambda=1,3$ at $D a=$ 0.001 . At $t=0.5$, isotherm contours increase smoothly from the bottom wall to approach the top wall.

Figures 20 and 21 present the average Nusselt number along the left and right wall of the cavity for different values of Darcy number $(D a=0.01,0.001)$ and viscosity ratio $\Lambda=1,3$. The average Nusselt number at the left (or right) wall has the same behavior for both viscosity ratio $\Lambda=1$ and 3 , at a particular Darcy number. Figure 20 shows that the average Nusselt number at the left (or right) wall first increases, then decreases, and finally attains steady solution for both viscosity ratios $\Lambda=1$ and 3 .

Figure 21a shows that the average Nusselt number at the left wall first decreases then increases and again decreases to attain the steady-state solution for both viscosity ratios $\Lambda=1$ and 3, at $D a=0.001$. The average Nusselt number at the right wall was first raised then reduced to achieve a steady-state solution (see Figure 21b). 


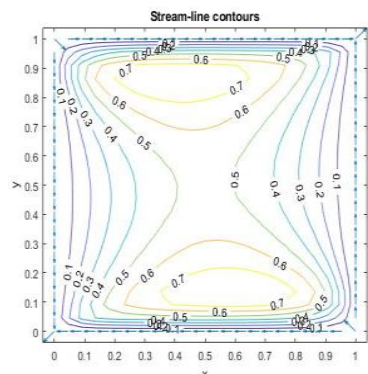

(a) $t=0.01$

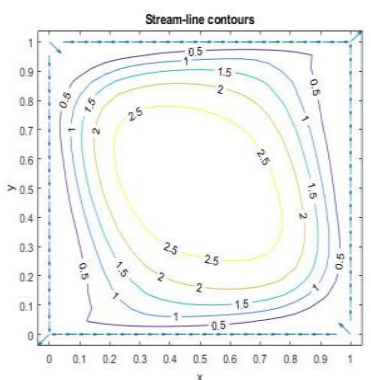

(b) $t=0.1$

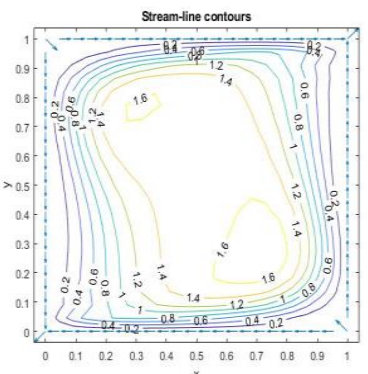

(c) $t=0.5$

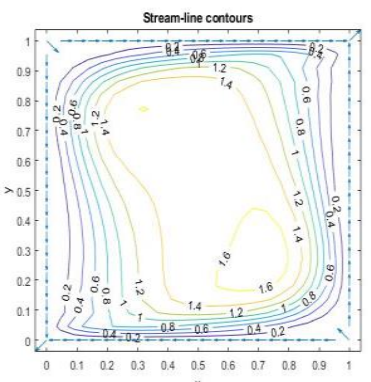

(d) $t=1,10$

Figure 12. Streamline contours for four-sided lid driven square porous cavity with $D a=0.01, \Lambda=1$

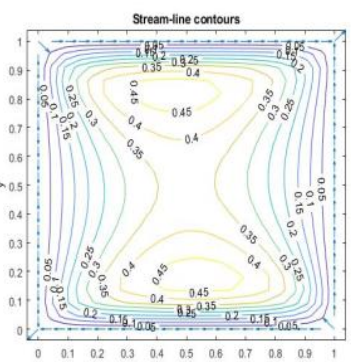

(a) $t=0.01$

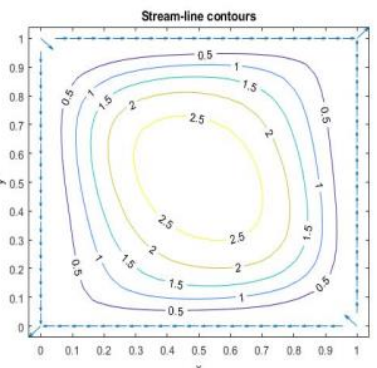

(b) $t=0.1$

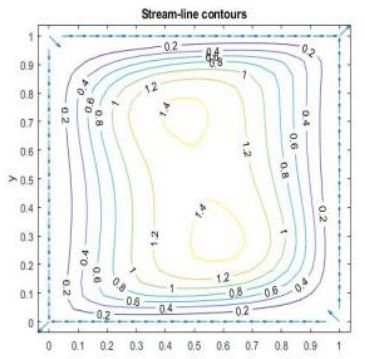

(c) $t=0.5$

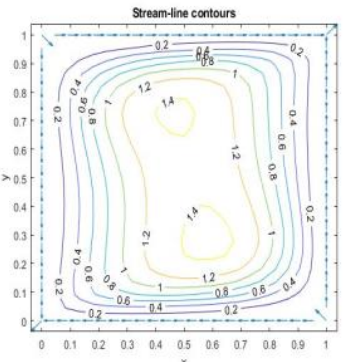

(d) $t=1,10$

Figure 13. Streamline contours for four-sided lid driven square porous cavity with $D a=0.01, \Lambda=3$

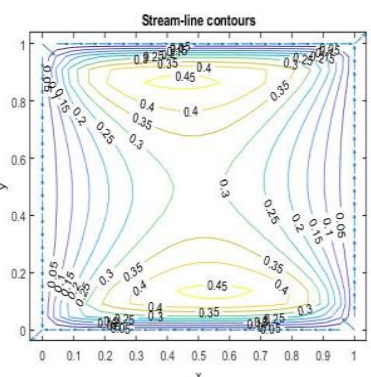

(a) $t=0.01$

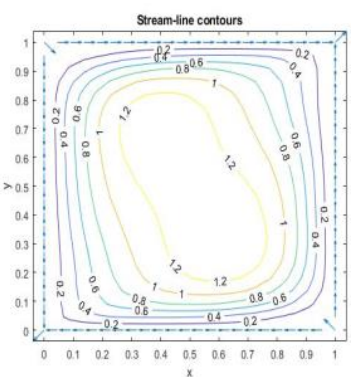

(b) $t=0.1$

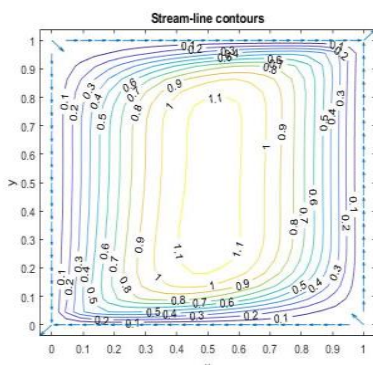

(c) $t=0.5$

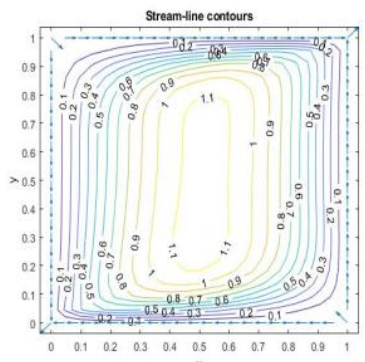

(d) $t=1,10$

Figure 14. Streamline contours for four-sided lid driven square porous cavity with $D a=0.001, \Lambda=1$

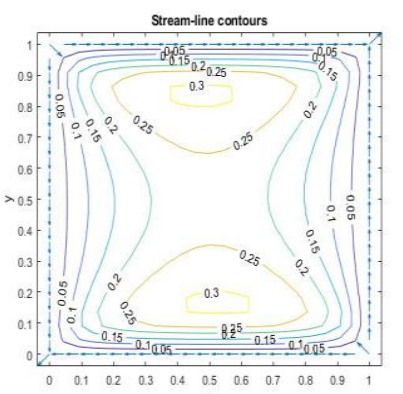

(a) $t=0.01$

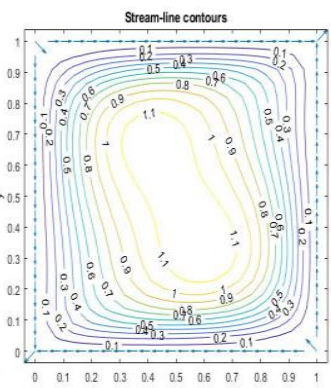

(b) $t=0.1$

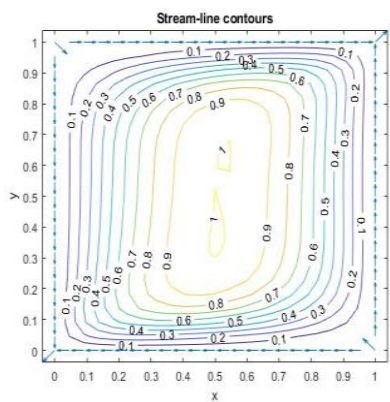

(c) $t=0.5$

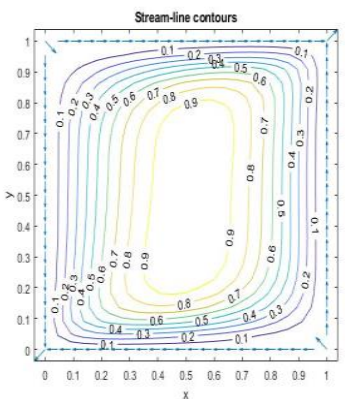

(d) $t=1,10$

Figure 15. Streamline contours for four-sided lid driven square porous cavity with $D a=0.001, \Lambda=3$

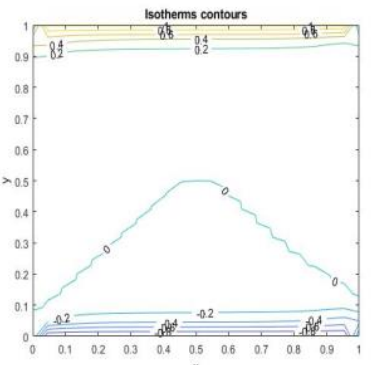

(a) $t=0.01$

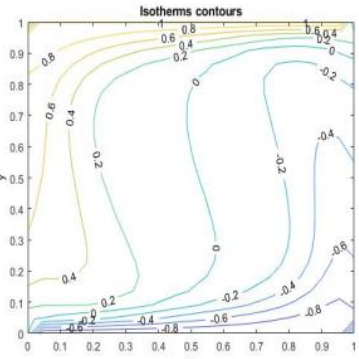

(b) $t=0.1$

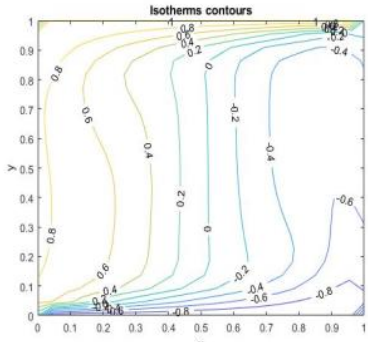

(c) $t=0.5$

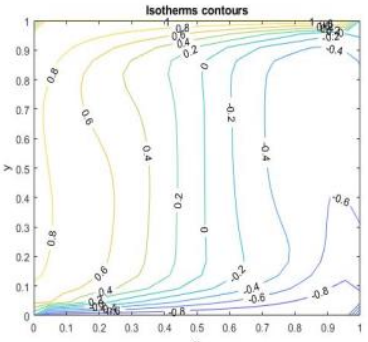

(d) $t=1,10$

Figure 16. Isotherms contours for four-sided lid driven square porous cavity with $D a=0.01, \Lambda=1$ 


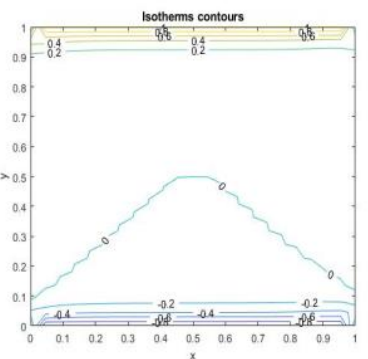

(a) $t=0.01$

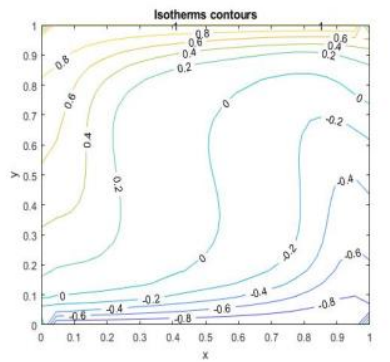

(b) $t=0.1$

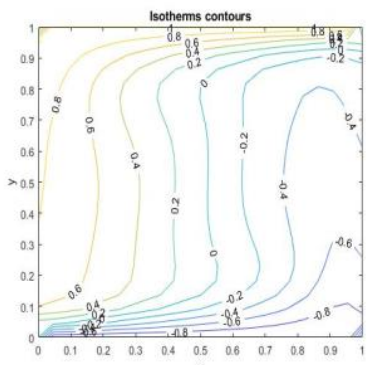

(c) $t=0.5$

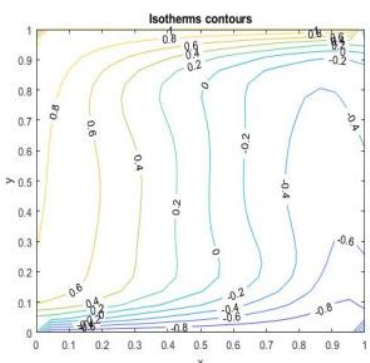

(d) $t=1,10$

Figure 17. Isotherms contours for four-sided lid driven square porous cavity with $D a=0.01, \Lambda=3$

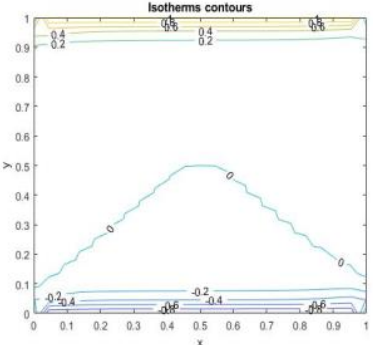

(a) $t=0.01$

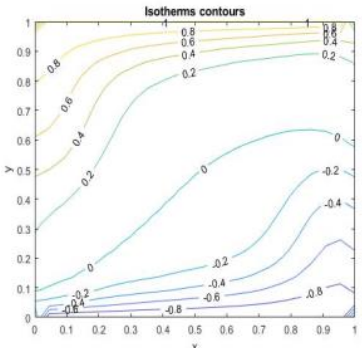

(b) $t=0.1$

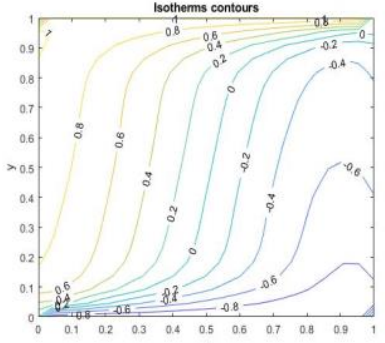

(c) $t=0.5$

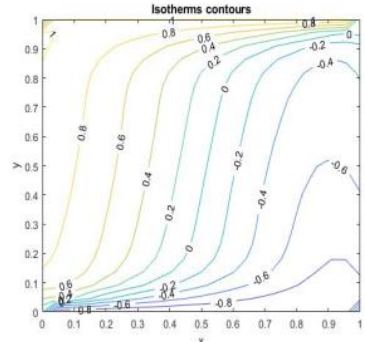

(d) $t=1,10$

Figure 18. Isotherms contours for four-sided lid driven square porous cavity with $D a=0.001, \Lambda=1$

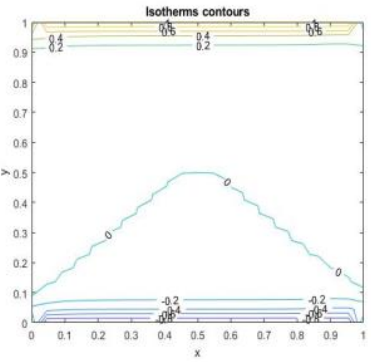

(a) $t=0.01$

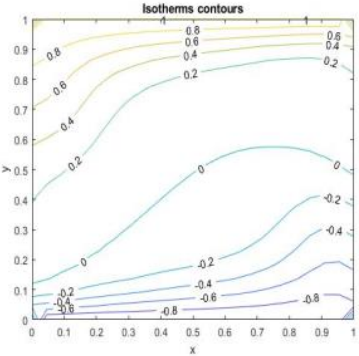

(b) $t=0.1$
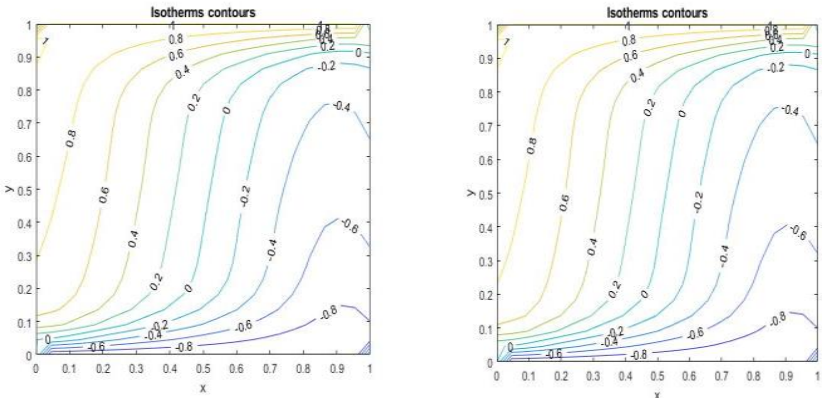

Figure 19. Isotherms contours for four-sided lid driven square porous cavity with $D a=0.001, \Lambda=3$

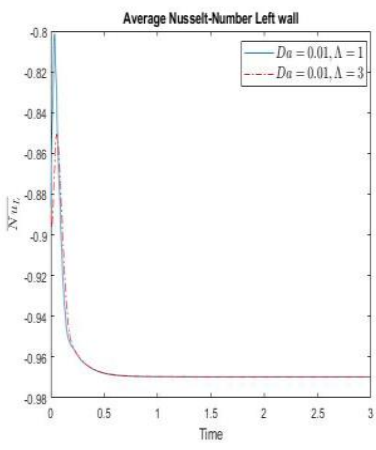

(a) Left Wall

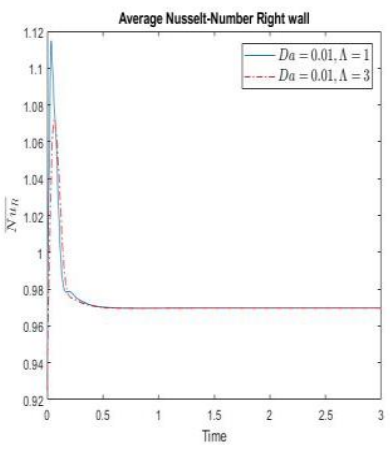

(b) Right Wall

Figure 20. Average Nusselt-Number for a four-sided lid-driven square porous cavity with $D a=0.01, \Lambda=1$ and 3 , at left and right wall

The average Nusselt number at the left (right) wall decrease with an increase of viscosity ratio from $\Lambda=1$ to 3 for both the Darcy number $(D a=0.01,0.001)$. It also decreases with a decrease of Darcy number from $D a=0.01$ to 0.001 at a particular viscosity ratio $(\Lambda=1$ and 3$)$.

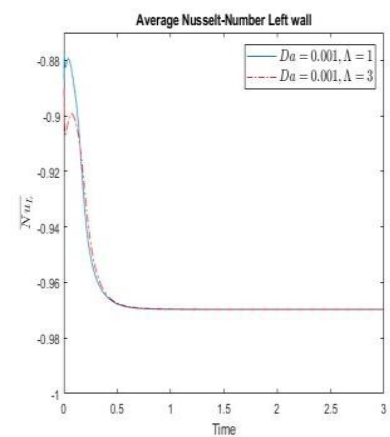

(a) Left Wall

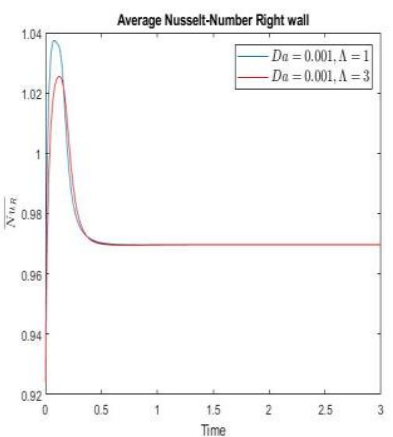

(b) Right Wall
Figure 21. Average Nusselt-Number for a four-sided lid-driven square porous cavity with $D a=0.001, \Lambda=1$ and 3 , at left and right wall

\section{CODE VALIDATION}

To validate our present computer code, we have compared the streamline contours and isotherm contours with the results of Venkatachalappa et al. [1], in which all walls are kept 
stationary. It has been found that our results and those of [1] are good in agreement (see Figures 22 and 23).

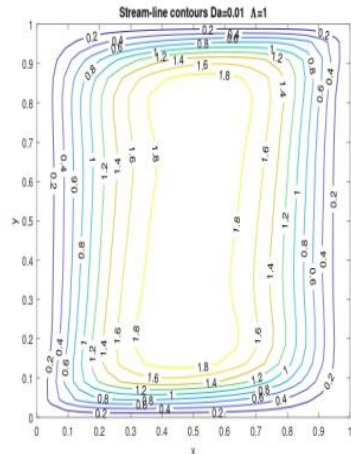

(a) $D a=0.01, \Lambda=1$

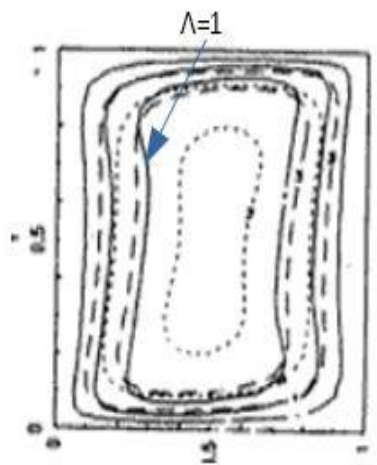

(c) $D a=0.01, \Lambda=1$

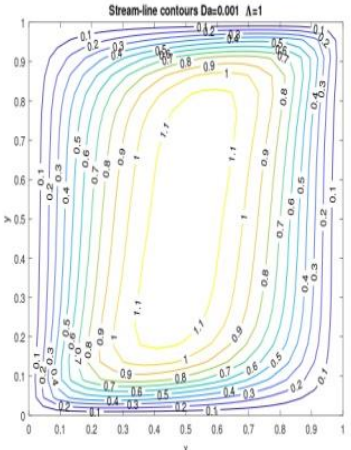

(b) $D a=0.001, \Lambda=1$

$\Lambda=1$

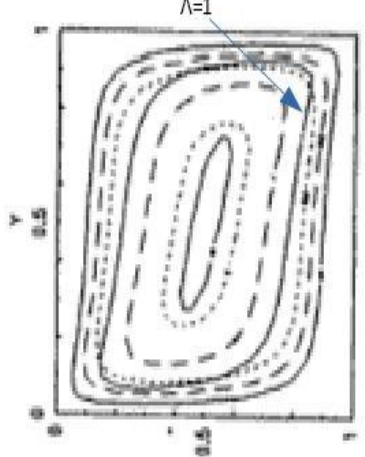

(d) $D a=0.001, \Lambda=1$
Figure 22. Comparison between the present result (top) and Venkatachalappa et al. [1] of streamline contours for $D a=$ $0.01,0.001$ with viscosity ratio $\Lambda=1$

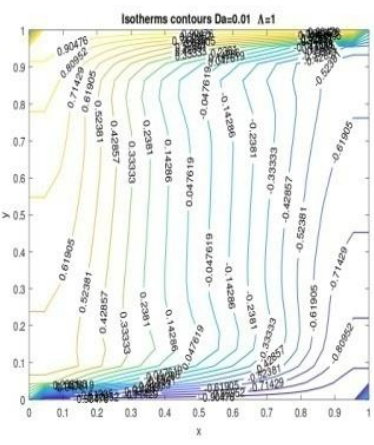

(a) $D a=0.01, \Lambda=1$

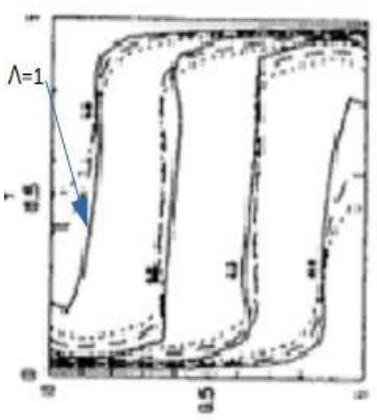

(c) $D a=0.01, \Lambda=1$

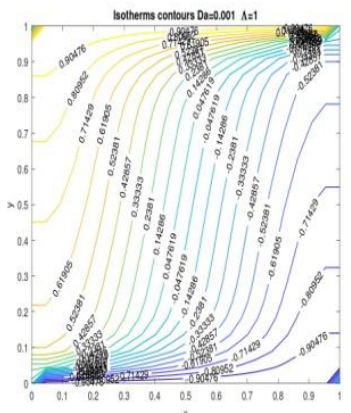

(b) $D a=0.001, \Lambda=1$ $\Lambda=1$

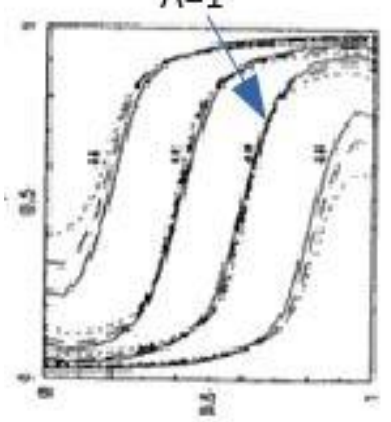

(d) $D a=0.001, \Lambda=1$
Figure 23. Comparison between the present result (top) and Venkatachalappa et al. [1] of isotherms contours for $D a$ $=0.01,0.001$ with viscosity ratio $\Lambda=1$

\section{CONCLUSIONS}

This paper investigates the mixed convection inside a twosided and four-sided lid-driven square cavity filled with porous media. We have employed the finite difference method to calculate the numerical solutions. We have compared our present results with the particular case in which all four walls of the cavity are stationary with previously published work and found to be in good agreement. We can conclude the following points from the current study.

\section{Case 1: Two-sided lid-driven square cavity}

I. The absolute value of $\psi$ decreases with an increase of viscosity ratio $\Lambda=1$ to 3 and a decrease of Darcy number $D a=0.01$ to 0.001 .

II. The viscosity ratio has a significant role in the numerical computation of isotherm contours at $D a=$ 0.01 . The effect of viscosity ratio $\Lambda=1,3$ is negligible with decreasing of Darcy number $D a=$ 0.01 to 0.001 .

III. The average Nusselt number at the left and right wall has oscillating behavior for $D a=0.01$ with viscosity ratio $\Lambda=1$ and 3 . The average Nusselt number at the left and right wall for $\Lambda=3$ attains an approximate steady-state solution at $t=1.25$.

\section{Case 2: Four-sided lid-driven square cavity}

I. The stream function $\psi$ decreases with an increasing of viscosity ratio $\Lambda=1$ to 3 and a decreases of Darcy number from $D a=0.01$ to 0.001 .

II. The absolute value of average Nusselt number at left (or right) wall decrease with an increase of viscosity ratio from $\Lambda=1$ to 3 and a decreasing of Darcy number from $D a=0.01$ to 0.001 .

\section{REFERENCES}

[1] Venkatachalappa, M., Shivakumara, I.S., Sankar, M., Prasanna, B.M.R. (1997). Natural convection in a rectangular porous cavity. The Seventh Asian Congress of Fluid Mechanics, pp. 561-564.

[2] Elaprolu, V., Das, M.K. (2009). Mixed convection in a buoyancy-assisted two-sided lid-driven cavity filled with a porous medium. International Journal of Numerical Methods for Heat and Fluid Flow, 19(3/4): 329-351. http://dx.doi.org/10.1108/09615530910938317

[3] Saeid, N.H., Mohamad, A.A. (2005). Natural convection in a porous cavity with spatial sidewall temperature variation. International Journal of Numerical Methods for Heat and Fluid Flow, 15(6): 555-566. http://dx.doi.org/10.1108/09615530510601459

[4] Mansour, M.A., Abd El-Aziz, M.M., Mohamed R.A., Ahmed S.E. (2011). Numerical simulation of natural convection in wavy porous cavities under the influence of thermal radiation using a thermal non-equilibrium model. Transport in Porous Media, 86: 585-600. http://dx.doi.org/10.1007/s11242-010-9641-5

[5] Basak, T., Roy, S., Paul, T., Pop, I. (2006). Natural convection in a square cavity filled with a porous medium: Effects of various thermal boundary conditions. International Journal of Heat and Mass Transfer, 49: 
$1430-1441$.

http://dx.doi.org/10.1016/j.ijheatmasstransfer.2005.09.0 18

[6] Badruddin, I.A., Zainal, Z.A., Aswatha Narayana, P.A., Seetharamu, K.N. (2007). Numerical analysis of convection conduction and radiation using a nonequilibrium model in a square porous cavity. International Journal of Thermal Sciences, 46: 20-29. http://dx.doi.org/10.1016/j.ijthermalsci.2006.03.006

[7] Chattopadhyay, A., Pandit, S.K. (2015). A Peclet number based analysis of mixed convection for lid-driven porous trapezoidal enclosure. Procedia Engineering, 127: 628635. http://dx.doi.org/10.13140/RG.2.1.1069.3206

[8] Kandaswamy, P., Muthtamilselvan, M., Lee, J. (2008). Prandtl number effects on mixed convection in a liddriven porous cavity. Journal of Porous Media, 11(8): 791-801.

[9] Mohan, C.G., Satheesh, A. (2016). The numerical simulation of double-diffusive mixed convection flow in a lid-driven porous cavity with magnetohydrodynamic effect. Arabian Journal for Science and Engineering, 41(5): 1867-1882. http://dx.doi.org/10.1007/s13369015-1998-x

[10] Md. Hidayathulla Khan, B., Ramachandra Prasad, V., Bhuvana Vijaya, R. (2018). Unsteady mixed convective flow in a porous lid-driven cavity with constant heat ux. In: Singh M., Kushvah B., Seth G., Prakash J. (eds) Applications of Fluid Dynamics, Lecture Notes in Mechanical Engineering, Springer, Singapore. https://doi.org/10.1007/978-981-10-5329-0_32

[11] Elaprolu, V., Das, M.K. (2007). Laminar mixed convection in a parallel two-sided lid-driven differentially heated square cavity filled with a fluidsaturated porous medium. Numerical Heat Transfer, Part A: $\quad$ Applications, 53(1): 88-110. http://dx.doi.org/10.1080/10407780701454006.

[12] Muthtamilselvan, M., Das, M.K., Kandaswamy, P. (2010). Convection in a lid-driven heat-generating porous cavity with alternative thermal boundary conditions. Transport in Porous Media, 8(2): 337-346. http://dx.doi.org/10.1007/s11242-009-9429-7

[13] Behzadi, T., Shirvan, K.M., Mirzakhan, S., Sheikhrobat, A.A. (2015). Numerical simulation on effect of porous medium mixed convection heat transfer in a ventilated square cavity. International Conference on Computational Heat and Mass Transfer, Procedia Engineering, 127: 221-228 http://dx.doi.org/10.1016/j.proeng.2015.11.333

[14] Mittal, N., Satheesh, A., Santhosh Kumar, D. (2014). Numerical simulation of mixed - convection flow in a lid-driven porous cavity using different nanofluids. Heat Transfer-Asian Research, 43(1): 1-16. http://dx.doi.org/10.1002/htj.21075

[15] Chattopadhyay, A., Pandit S.K., Sarma, S.S., Pop, I. (2016). Mixed convection in a double lid-driven sinusoidally heated porous cavity. International Journal of Heat and Mass Transfer, 93: 361-378. http://dx.doi.org/10.1016/j.ijheatmasstransfer.2015.10.0 10

[16] Md. Hidayathulla Khan, B., Prasad, V.R., Vijaya, R.B. (2018). Thermal radiation on mixed convective flow in a porous cavity: Numerical Simulation. Nonlinear Engineering, 7(4): http://dx.doi.org/10.1515/nleng-2017-0053
[17] Vusala, A., Kumar, M. (2017). Numerical solutions of 2$\mathrm{d}$ unsteady incompressible flow with heat transfer in a driven square cavity using stream function-vorticity formulation. International Journal of Heat and Technology, 35(3): 459-473. http://dx.doi.org/10.18280/ijht.350303

[18] Bagai, S., Kumar, M., Patel, A. (2020). The four-sided lid driven square cavity using stream function-vorticity formulation. Journal of Applied Mathematics and Computational Mechanics, 19(2): 17-30. https://doi.org/10.17512/jamcm.2020.2.02

[19] Bagai, S., Kumar, M., Patel, A. (2020). Mixed convection in four-sided lid-driven sinusoidally heated porous cavity using stream function-vorticity formulation. SN Applied Sciences, 2: 2066. https://doi.org/10.1007/s42452-020-03815-7

[20] McDonough, J.M. (2007). Lectures on computational numerical analysis of partial differential equations. available from the website http://www.engr.uky.edu/ acfd.

[21] Smith, G.D. (1985). Numerical Solution of Partial Differential Equations: Finite Difference Methods. Oxford University Press, New York, U.S.A.

\section{NOMENCLATURE}

$\begin{array}{ll}D a & \text { Darcy number } \\ g & \text { Acceleration due to gravity } \\ G r & \text { Grashof number } \\ \mathrm{K} & \text { thermal conductivity, } \mathrm{W} \cdot \mathrm{m}^{-1} \cdot \mathrm{K}^{-1} \\ L & \text { Length of the cavity } \\ n & \text { Number of grid points } \\ \overline{N u} & \text { Average Nusselt number } \\ P & \text { Dimensionless pressure } \\ P r & \text { Prandtl number } \\ \operatorname{Re} & \text { Reynolds number } \\ S & \text { Ratio of specific heat } \\ T & \text { Dimensionless temperature } \\ \theta & \text { Dimensional Temperature } \\ \theta_{0} & \text { Reference Temperature } \\ t & \text { Dimensionless Time } \\ t^{\prime} & \text { Dimensional Time } \\ u, v & \text { Dimensionless Component of velocity } \\ u^{\prime}, v^{\prime} & \text { Dimensional Component of velocity } \\ x, y & \text { Dimensionless Cartesian Co-ordinates } \\ x^{\prime}, y^{\prime} & \text { Dimensional Cartesian Co-ordinates } \\ \Delta x, \Delta y & \text { Grid spacing along } x \text { and } y \text { axis. } \\ \Delta \mathrm{t} & \text { Grid spacing along } t \text {-direction }\end{array}$

\section{Greek symbols}

$\begin{array}{ll}\alpha & \text { thermal diffusivity, } \mathrm{m}^{2} \cdot \mathrm{s}^{1} \\ \beta & \text { thermal expansion coefficient, } \mathrm{K}^{-1} \\ \Lambda & \text { viscosity ratio } \\ \theta & \text { dimensionless temperature } \\ \nu & \text { kinematic viscosity } \\ \Omega & \text { Dimensional vorticity } \\ \xi & \text { Dimensionless vorticity } \\ \lambda & \text { Eigenvalues of matrix } \\ \Psi & \text { Dimensionless stream function } \\ \Psi^{\prime} & \text { Dimensional stream function } \\ \varepsilon & \text { Porosity } \\ \mu & \text { Dynamic viscosity }\end{array}$


Subscripts

L

Left wall

Right wall

Grid points 